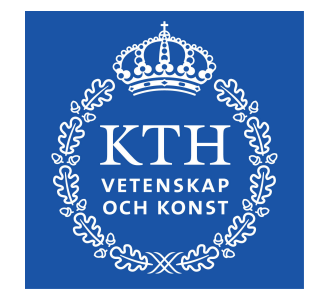

DEGREE PROJECT IN INFORMATION AND COMMUNICATION TECHNOLOGY,

SECOND CYCLE, 30 CREDITS

STOCKHOLM, SWEDEN 2020

\title{
Improved Precision Time Protocol with Relative Clock Phase Information
}

\author{
AVNEESH VYAS
}




\section{Abstract}

A common time reference among nodes is one of the key requirements in telecommunication, distributed control systems and industrial automation systems. For instance, 3 GPP LTE TDD standard requires at least $\pm 1.5 \mu$ sec time accuracy among base stations in order to resolve uplink and downlink transmission. Certain other emerging technologies such as wireless positioning, coordinated antenna beamforming have far more stringent timing requirements often in the order of sub-nanoseconds. For example, in wireless positioning method such as LTE OTDOA [1], every nano-second loss of precision translates into approximately $30 \mathrm{~cm}$ of positioning estimation error. In modern packet switched backhaul networks, time distribution protocols are used to distribute timing information from high quality clock source to network nodes. The accuracy and precision of the time distribution protocol improve if it runs as close to hardware as possible so that variable software queuing delays are reduced or eliminated. IEEE 1588-2008 Precision Time Protocol, PTP with hardware timestamping promises higher precision compared to purely software based protocols. However, network asymmetry, variable queuing delays, and timestamping errors in underlying transport, limit the highest time synchronization precision of most commercial PTP deployments to a few hundred nanoseconds. In this work, the operation and estimation capability of IEEE 1588-2008 Precision Time Protocol (PTP) is formally analysed and PTP improvement in the form of super-imposed clock timing aware signal exchange protocol, is proposed. The proposed protocol operating alongside PTP provides independent clock parameter estimates without impacting any existing PTP infrastructure. In addition, it provides relative clock phase offset estimate which is otherwise not detectable through standard PTP. Furthermore, this work through qualitative and quantitative analysis, demonstrates how the supplementary estimates from the proposed method can be used to improve overall clock synchronization accuracy. 


\section{Sammanfattning}

En gemensam tidsreferens bland noder är ett av de viktigaste kraven för telekommunikation, distribuerade styrsystem och industriella automatiseringssystem. Till exempel kräver 3GPP LTE TDD-standarden minst $\pm 1.5 \mu \mathrm{sec}$ mikrosekunders tidsnoggrannhet mellan basstationer för att lösa upp- och nedlänksöverföring. Vissa andra framväxande tekniker som trådlös positionering och koordinerad antennstrålformning har mycket strängare tidskrav, ofta i storleksordningen sub-nanosekunder. Till exempel, i den trådlösa positioneringsmetoden LTE OTDOA [1], översätts varje nanosekund i förlust av precision till ungefär $30 \mathrm{~cm}$ positioneringsfel. I moderna paketkopplade anslutningsnätverk används tidsfördelningsprotokoll för att distribuera tidsinformation från en högklassig klock-källa till nätverksnoder.

Tidsfördelningsprotokollets noggrannhet och precision förbättras om det körs så nära hårdvaran som möjligt så att fördröjningar i variabla mjukvaruköer minskas eller elimineras. IEEE 1588-2008 Precision Time Protocol (PTP) med hårdvarutidsstämpling lovar en högre precision jämfört med rent mjukvarubaserade protokoll. Nätverksasymmetri, variabla köfördröjningar och tidsstämplingsfel i underliggande transport begränsar dock den högsta tidssynkroniseringsprecisionen för de flesta kommersiella PTP driftsättningarna till några hundra nanosekunder. I detta arbete analyseras driften och skattningsförmågan för IEEE 1588-2008 PTP formellt och en PTP-förbättring i form av ett påtvingat klocktidsmedvetet signalutbytesprotokoll föreslås. Det föreslagna protokollet som fungerar tillsammans med PTP ger oberoende klockparameterskattningar utan att påverka någon befintlig PTP infrastruktur. Dessutom ger den en relativ skattning av klockfas som annars inte kan detekteras genom standard PTP. Dessutom visar detta arbete genom kvalitativ och kvantitativ analys hur de kompletterande skattningarna från den föreslagna metoden kan användas för att förbättra den totala noggrannheten i klocksynkroniseringen. 


\section{Acknowledgements}

I would like to express my deepest gratitude to my thesis supervisor, Dr. Satyam Dwivedi (Ericsson Research) whose extensive knowledge, guidance, and relentless encouragement made this work possible. I am also grateful to the Network Sync and Interconnect (NSI) department at Ericsson AB and KTH's School of Electrical Engineering And Computer Science, for providing necessary resources for this project. Finally, I would like to thank my daughters, Aditi and Anika and my wife, Deepa for the unwavering love and support. 


\section{Publication and Patents}

This thesis work has resulted in the following publication and patent applications:

1. Vyas, Avneesh, Satyam Dwivedi, and Fredrik Gunnarsson. "Improved Precision Time Protocol with Relative Clock Phase Information." 2018 IEEE International Symposium on Precision Clock Synchronization for Measurement, Control, and Communication (ISPCS). IEEE, 2018.

2. Satyam Dwivedi, Bo Hagerman, Fredrik Gunnarsson, Avneesh Vyas. "A method and a first device for clock synchronization."

Patent WO2019177503A1, March 14, 2018

3. Satyam Dwivedi, Fredrik Gunnarsson, Avneesh Vyas, Magnus Sandgren. "Methods, second node and apparatus for determining clock asynchronization." Patent WO2020067941A1, September 27, 2018 


\section{Contents}

1 Introduction 9

1.1 Background . . . . . . . . . . . . . . . . . 9

1.2 Clock Synchronization . . . . . . . . . . . . . . . . 12

1.3 Types of Synchronization . . . . . . . . . . . . . . . . . 12

1.3.1 Frequency Synchronization . . . . . . . . . . . . . . 12

1.3.2 Phase Synchronization . . . . . . . . . . . . . . . . 12

1.3.3 Time Synchronization . . . . . . . . . . . . . 13

1.4 Existing Methods for Clock Synchronization . . . . . . . . . 14

1.4.1 Logical Clock Synchronization . . . . . . . . . . . . . 14

1.4.2 Physical Clock Synchronization . . . . . . . . . . . . 15

2 Clock Model for PTP Analysis 18

2.1 Clock Model . . . . . . . . . . . . . . . . . . . . . . 18

2.1.1 Single Clock Model . . . . . . . . . . . . . . . . . . 18

2.1.2 Relative Clock Model . . . . . . . . . . . . . . . . . . . 19

2.1.3 Synchronization Objective . . . . . . . . . . . . . 21

2.2 Precision Time Protocol . . . . . . . . . . . . . . . . 21

2.2 .1 Operation . . . . . . . . . . . . . . . . 21

2.2 .2 Estimation . . . . . . . . . . . . . . . . . 22

2.2 .3 Limitations . . . . . . . . . . . . . . . . . . 23

3 Proposed Improvement 24

3.1 Timestamp Resolution Error or Unknown Clock Phase Offset . 24

3.1.1 Round Trip Time (RTT) Based Clock Parameter Estimation . . . . . . . . . . . . . . . 25

3.2 Proposed PTP Improvements . . . . . . . . . . . . . . . . 28

3.2.1 Improved Two-way Precision Time Protocol . . . . . . 28

3.2.2 Improved One-way Precision Time Protocol . . . . . . 30 
4 Simulations and Results 34

4.1 Simulation Methodology . . . . . . . . . . . . . . . 34

4.2 Simulation Configuration Parameters . . . . . . . . . . . 35

4.3 Results and Analysis - Improved Two-way PTP . . . . . . . . 36

4.4 Results and Analysis - Improved One-way PTP . . . . . . . . 40

5 Comparison with White Rabbit 44

5.1 White Rabbit . . . . . . . . . . . . . . . . . . 44

5.2 RTT based PTP improvement . . . . . . . . . . . . 45

6 Discussion and Conclusion 47

$\begin{array}{ll}\text { Bibliography } & 49\end{array}$ 


\section{List of Figures}

1.1 Frequency Synchronization $[2] \ldots \ldots$. . . . . . . . . . . 13

1.2 Phase Synchronization $[2] \ldots \ldots$. . . . . . . . . . . . . . 13

1.3 Time Synchronization $[2] \ldots \ldots$. . . . . . . . . . . 14

2.1 Relative Clock Model. . . . . . . . . . . . . . . . 20

2.2 A Simplified PTP Timestamp Exchange. . . . . . . . . . . . 22

3.1 Round Trip Time Sample as described in [3] . . . . . . . . . 26

3.2 Round Trip Time Sawtooth . . . . . . . . . . . . . . . . 26

3.3 RTT Measurement Protocol. . . . . . . . . . . . . . . 28

3.4 Frequency and Phase Estimation through One-Way PTP. . . . 32

4.1 Two-way sync error $\left[f_{d}=100 \mathrm{~Hz}\right.$, path delay std_dev $\left.=5 n s\right] \quad . \quad 37$

4.2 Two-way sync error $\left[f_{d}=100 \mathrm{~Hz}\right.$, path delay std_dev $\left.=10 \mathrm{~ns}\right] \quad 37$

4.3 Two-way sync error $\left[f_{d}=150 \mathrm{~Hz}\right.$, path delay std_dev $\left.=5 \mathrm{~ns}\right] .38$

4.4 Two-way sync error $\left[f_{d}=150 \mathrm{~Hz}\right.$, path delay std_dev $\left.=10 \mathrm{~ns}\right] \quad 38$

4.5 Two-way sync error $\left[f_{d}=200 \mathrm{~Hz}\right.$, path delay std_dev $\left.=5 n s\right] \quad . \quad 39$

4.6 Two-way sync error $\left[f_{d}=200 H z\right.$, path delay std_dev $\left.=10 n s\right] \quad 39$

4.7 One-way sync error $\left[f_{d}=100 \mathrm{~Hz}\right.$, path delay std_dev $\left.=5 n s\right] .41$

4.8 One-way sync error $\left[f_{d}=100 \mathrm{~Hz}\right.$, path delay std_dev $\left.=10 \mathrm{~ns}\right] .41$

4.9 One-way sync error $\left[f_{d}=150 \mathrm{~Hz}\right.$, path delay std_dev $\left.=5 n s\right] .42$

4.10 One-way sync error $\left[f_{d}=150 \mathrm{~Hz}\right.$, path delay std_dev $\left.=10 \mathrm{~ns}\right] .42$

4.11 One-way sync error $\left[f_{d}=200 \mathrm{~Hz}\right.$, path delay std_dev $\left.=5 n s\right] .43$

4.12 One-way sync error $\left[f_{d}=200 H z\right.$, path delay std_dev $\left.=10 n s\right] .43$

5.1 White Rabbit Synchronization Scheme [4] . . . . . . . . . 46 


\section{Chapter 1}

\section{Introduction}

This chapter provides necessary background knowledge, goal and methodology followed for the thesis work. In the last sub-section of this chapter, the structure of this report is presented.

\section{$1.1 \quad$ Background}

A common time reference among disjoint nodes is one of the key requirements of modern communication networks. Traditionally, time synchronizing base stations was less of a problem as synchronization information was deducible from the time division multiplexing (TDM) based circuit-switched physical link. In other words, the receiving node could simply tune its internal oscillator to the bit-rate of incoming traffic from the backhaul. But, recent move towards IP/Ethernet in telecom backhaul all the way up to radio equipment (RE), mainly to avail benefits of statistical multiplexing as in 5G, has diminished the traditional synchronization information source. This has forced operators and vendors to employ alternate methods to synchronize network nodes to the common time reference.

Theoretically, Global Positioning System (GPS) [5] receiver at all base stations can allow time locking to a common reference in a simple and straightforward manner. Unfortunately, GPS signal is weak and even unavailable in several settings such as indoors or in tunnels. Consequently, in practice only a select few nodes with suitable conditions are attached to a high-quality time source such as GPS. These nodes acting as clock masters then distribute timing information to one or more slave nodes. This 
is where, time distribution protocols such as IETF Network Time Protocol (NTP) [6] and IEEE 1588-2008 Precision Time Protocol (PTP) [7] play a major role. Among these two master-slave protocols, PTP with physical layer timestamping, is preferred when the desired timing accuracy is in the order of micro seconds or less. 3GPP's Long Term Evolution TDD standard requires at least $\pm 1.5 \mu \mathrm{sec}$ timing accuracy among base stations to detect start of frame boundaries and avoid interference between uplink and downlink timeslots. And therefore, PTP is the natural choice for synchronizing LTE TDD networks [8].

Although PTP promises very high precision but its real performance depends on its implementation and network conditions. With its potential error sources, achieving a finer time accuracy precision than a few hundred nanoseconds without cost escalation is a challenge in most commercial PTP deployments. At the same time, several emerging technologies such as wireless positioning are sensitive to even small timing errors and therefore pose far stringent timing requirements, often in the order of sub-nanoseconds. For instance, in LTE's Observed Time Difference of Arrival (OTDOA) based positioning, every nano-second loss of time synchronization among participating base station translates into approximately $30 \mathrm{~cm}$ of positioning uncertainty of the user equipment. Furthermore, 5G aims to provide higher traffic volume, improved indoor coverage and advanced features such as carrier aggregation and coordinated antenna beam-forming which shall require even more stringent timing constraints. Consequently, 5G standard is expected to reduce the time error budget to $\pm 130 \mathrm{~ns}[9]$. Other domains such as distributed control system, location aware industrial automation systems, financial and scientific networks also have several applications that require near to perfect time synchronization. Consequently, there is a definite need to improve existing time synchronization methods.

To improve PTP's time accuracy, CERN's White Rabbit (WR) project has attempted to reduce the effect of PTP's two most dominant error sources viz. unknown network asymmetry and limited timestamp resolution [4]. They have proposed an extension to PTP, in the form of a PTP profile, which through additional signaling enables accurate determination of physical link asymmetry and relative clock phase difference between PTP peers. The former is used to accurately estimate the mean path delay of PTP messages whereas the latter is used to improve the timestamp resolution. WR has demonstrated sub-nanosecond time accuracy between WR compliant SyncE transport switches connected over a $5 \mathrm{~km}$ fiber optic link. Although this is a 
remarkable development, but the proposed WR PTP extension requires specific network transport and compliant switches with hardware phase locked loop capability. This can be seen as a significant impediment in WR's widespread adoption. For instance, replacing existing switches may not be financially viable for many telecommunication operators interested in high timing accuracy. For them, the new improvement should be unobtrusive enough to be applied without major hardware upgrades. Also, as sync signal may have to traverse through multiple transport network technologies, any dependency on specific transport may not be desirable in the context of telecommunication networks.

Dwivedi et al. [3], have devised a Round Trip Time (RTT) based clock synchronization method to precisely estimate clock parameters between two wireless nodes without any timestamp exchange. Here, RTT measurements have been shown to follow a time varying periodic sawtooth behavior. This phenomenon results from the relative clock phase offset, $\phi$ and frequency offset, $f_{d}$ between master and slave nodes, which respectively determine the phase and the frequency of the sawtooth waveform. This work also provides several estimators to extract phase and frequency offsets from the RTT sawtooth measurement plot with different degrees of accuracy.

This thesis work presents and analyses an improved version of PTP that combines [3]'s clock timing aware RTT based method with standard PTP message exchange. The super-imposed protocol working alongside PTP provides independent clock parameter estimates in addition to those already provided by PTP. Having two independent estimates provides the possibility to fuse them to produce improved clock parameter estimates resulting in improved clock synchronization. In addition, the method also allows estimation of clock phase offset which is not possible through PTP. The determined clock phase, similar to White Rabbit's method [4], can be used to improve the timestamp precision beyond the resolution permitted by the clock frequency.

The rest of the report is structured as follows: This chapter presents the background and the overview of the state-of-the-art clock synchronization methods. Chapter 2 presents the standard clock model that can be used to formally analyze various time sync methods. The same chapter also analyses the estimation capability of PTP. Chapter 3 presents the proposed improvements to PTP and mathematically explains how it can be used to improve overall clock parameter estimation accuracy. Chapter 4 presents the simulation experiments and performance results of the evaluation of the described methods. Chapter 5 presents a comparison between White Rabbit's phase 
estimation and proposed RTT based clock phase estimation. Finally, chapter 6 provides the conclusion.

\subsection{Clock Synchronization}

Clock Synchronization among independent nodes is an essential requirement in several domains such as financial networks, scientific applications, distributed control systems, power distribution, telecommunication networks, robotics, aviation technology, space exploration and many more.

Clock synchronization improves efficiency and cost savings in certain domains such as power distribution and in others, it acts as the essential en-

abler for coordinated features such as Time Division Duplexing (TDD) of LTE radio channels, or sub-atomic particle detection in Large Hadron Collider (LHC) at CERN [10]. Through clock synchronized coordinated actions, automated systems can reduce wastage and thus operate in an environment friendly manner.

\subsection{Types of Synchronization}

In literature [2], the term 'clock synchronization' may mean one of the following types of clock synchronization.

\subsubsection{Frequency Synchronization}

Two clocks are frequency synchronized (or sometimes called syntonized) if their clock events are repeated at equal interval or in other words the two clocks have identical rate of their clock events. During the observation window, the clock events may not occur at the same time instance. Figure 1.1 shows two syntonized clocks with their respective events in two different colors [2].

\subsubsection{Phase Synchronization}

Two syntonized clocks are phase synchronized if their clock events occur at the same time instance. Notably, the clock do not need to have a common time origin to be phase synchronized. In other words, the two clocks may 
show different time-of-day (ToD) while still synchronized in phase. Figure 1.2 shows two phase synchronized clocks [2].

\subsubsection{Time Synchronization}

Finally, the two clocks are time synchronized if they show the same timeof-day at all time instances during the observation window. Time synchronization is achieved by first synchronizing frequency and phase and then adjusting time origin of one clock to match that of the other. In other words, time synchronization is an outcome of frequency and phase synchronization. In many contexts, the terms time and phase synchronization respectively are used interchangeably. Figure 1.3 shows two time synchronized clocks [2].

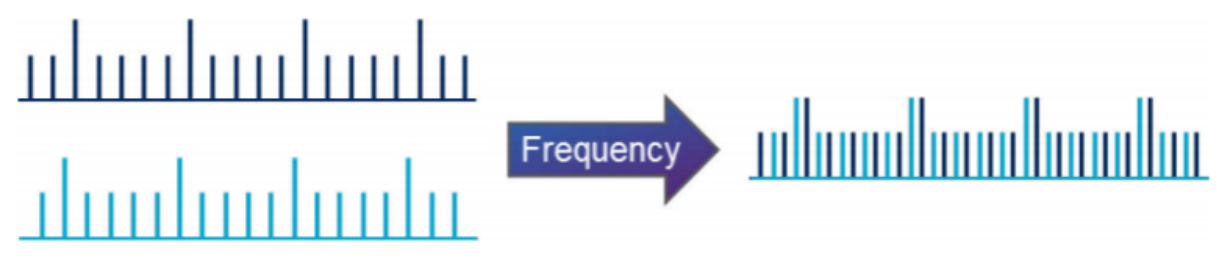

Figure 1.1: Frequency Synchronization [2]

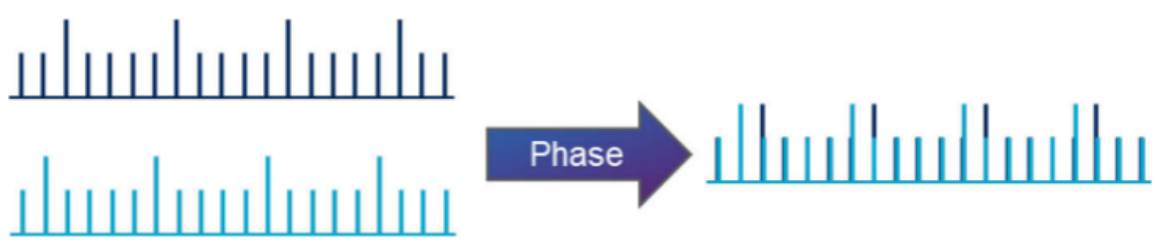

Figure 1.2: Phase Synchronization [2] 


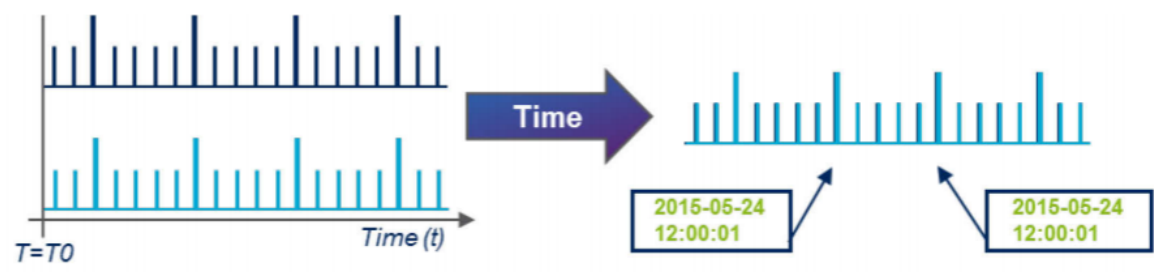

Figure 1.3: Time Synchronization [2]

\subsection{Existing Methods for Clock Synchroniza- tion}

In this section, a brief overview of the synchronization state-of-the-art is presented.

\subsubsection{Logical Clock Synchronization}

Certain networked systems such as distributed storage systems are expected to maintain global ordering of events occurring in the system. For instance, to ensure sequential consistency, a distributed key-value storage systems requires that all read and write operations are processed by all participant nodes in an identical order. If all nodes are perfectly time synchronized and all events are accurately timestamped, then ordering events is straightforward. But it is near to impossible to achieve perfectly synchronized network. Alternatively, Lamport proposed a scheme where events can be ordered based on physical causality which acts as a logical clock. In this Lamport logical clock system, each node (or process, as sometimes called in the context of distributed systems) maintains two logical timestamps- a logical local timestamp that is incremented before each local event, and a logical global timestamp that tracks the latest global event seen by the node in the form of an incoming message. This way, a node can determine 'happened-before' relation between events, and thereby order them accordingly. Vector clocks algorithm used to order events across several nodes in a distributed system, is also based on logical clocks. Amazon's Dynamo uses Lamport's logical clock to order database operations [11]. 


\subsubsection{Physical Clock Synchronization}

Communication systems have more stringent synchronization requirements than simple ordering of events. For example, LTE TDD base stations can at maximum be $\pm 1.5 \mu \mathrm{sec}$ off to each other to avoid overlapping of uplink and downlink subframes from neighboring base stations. There are several other feature such as LTE's OTDOA positioning that require time-of-day synchronization and therefore logical clocks may not be enough. Explained below are some techniques that enable time distribution among disjoint nodes.

\section{Global Navigation Satellite System (GNSS)}

GNSS is a constellation of several satellites orbiting around earth which working in a coordinate manner provides geo-spatial positioning capabilities through a hand-held GNSS receiver. The satellites are time synchronized to each other and use extremely precise atomic clocks which are traceable to Coordinate Universal Time (or UTC). A typical GNSS receiver, in addition to location information can also provide 1-Pulse-Per-Second (1PPS) signal and absolute time-of-the-day. Thus a node with an attached GNSS receiver can use the 1 PPS and time-of-the-day readings to frequency and time synchronize its internal clock to an external clock reference. Due to the ubiquitous nature of GNSS based systems, this method is considered to be the most straightforward method to time synchronize geographically distributed nodes of a network. Unfortunately, there are certain limitations which do not let GNSS become the universal method for clock sync. For instance, this method requires view of the satellites and therefore cannot be used indoors or in tunnel. Additionally, GNSS radio signal is prone to multipath fading occurring due to reflections from buildings, and other terrestrial objects which further contributes to timing inaccuracies [5].

These limitations necessitates the need of time distribution protocols (discussed in the following sections) which can basically transfer timing information from node with high quality clock source (say, GNSS) to other nodes

using the data network. Consequently, they eliminate the need of GNSS receiver on all nodes.

\section{Network Timing Protocol (NTP)}

NTP is a timestamp exchange based network layer protocol to distribute timing across packet switched data network. The latest protocol version 
(NTPv4) is specified in IETF RFC 5905 [6]. The protocol works in a client server mode where the NTP client periodically sends query messages to several NTP servers which in turn send thier responses to the client. In the process, all incoming and outcoming packets are timestamped and the values are included in the respective messages. Using the timestamps, the client can calculate the absolute time difference between the NTP server and local clock and synchronize its local clock to that of one of the NTP servers. NTP's performance is adversely affected by network asymmetry (unequal forward and return routes), and packet delay variations due variable queuing or routing delays at intermediate nodes. One to few hundred milliseconds time accuracy depending upon the network conditions [12].

\section{Precision Timing Protocol (PTP)}

IEEE 1588-2008 PTP [7] is a time distribution protocol that is capable of delivering significantly higher timing accuracy (in the order of $20 n s-10 \mu \mathrm{sec}$ ) compared to that of NTP. It does so by relying on hardware based timestamping units (TSU) instead of software timestamping used by NTP. Hardware TSU enables significantly more accurate timestamping of egress/ingress PTP time packets. Further, IEEE 1588 standard also specifies the use of PTP compliant hardware on intermediate switches in the form of transparent clocks. Transparent clock measures the resident time of each PTP packet in the switch and adds it to the PTP correction field of the packet before forwarding it to the next switch. The end node can then compensate the time measurements for the total resident time in switches. This significantly improves the end-to-end precision of each time measurement. Given the high precision attainable by PTP, it has become the default choice for time distribution in time-sensitive networks such as mobile networks, power grids, and scientific networks. As this thesis proposes improvements to PTP, a more detailed explanation of PTP's capability is in order, which is presented in 2.2 .

\section{Synchronous Ethernet (SyncE)}

Ethernet, traditionally used on LAN is fast replacing backhaul transport streaming protocols such as SONET/SDH. One undesired side effect of this development is that node receiving Ethernet traffic cannot use incoming Eth-

ernet bitrate as a source of clock synchronization which it could do previously 
with synchronous protocols such as SDH. Recognizing this weakness, ITUT specified SyncE standard that allows transfer of clock signal traceable to some high quality clock source, over Ethernet physical layer. It is important to note that to maintain clock synchronization using SyncE between two edge nodes of the network, all intermediate nodes should be SyncE compliant.

ITU-T G.8275.1 standard [13] defines the telecom PTP profile for full timing support that recommends using PTP over SyncE transport. In this mode, SyncE provides frequency synchronization and PTP provides time synchronization between the two node. 


\section{Chapter 2}

\section{Clock Model for PTP Analysis}

This chapter first introduces a clock model in 2.1 and then presents a formal analysis of PTP's performance in 2.2.

\subsection{Clock Model}

To formally analyze and compare time synchronization methods, a model representing a typical clock is presented in this section. This model has been used to describe clock behavior in [1] and [14].

\subsubsection{Single Clock Model}

A clock can be visualized as a device that measures time by counting clock edges or events separated by fixed time duration clock periods. Thus, a clock value, $C$ at any instance can be represented as

$$
C=n T+\beta,
$$

where $n$ is the number of clock edges with time period, T. $\beta$ is the initial clock phase at the start of the observation window.

Commercially, clocks are manufactured using electronic oscillator circuits that produce electric pulse at a particular frequency using the mechanical resonance of vibrating crystal of piezoelectric material such as quartz. The quartz crystal are formed to behave like an RLC circuit with precise resonant frequency. Thus it is more natural to express clock value in terms of the 
resonating or nominal frequency of its oscillator as follows

$$
C=\frac{n}{f_{0}}+\beta
$$

where $f_{0}$ is the nominal frequency of the clock.

However, the oscillator's actual operating frequency may be slightly different from its nominal frequency due to environmental conditions such as temperature that affects crystal oscillators. So, one may observe fractional deviation in the clock frequency vis-a-vis its nominal frequency. In the clock model, this fractional deviation in frequency can be incorporated by introducing an additional parameter, $\alpha=\frac{f_{0}}{f}$ where $f$ is the actual frequency of the oscillator during the observation window. Thus, the revised clock model can be expressed as

$$
C=\alpha \frac{n}{f_{0}}+\beta
$$

where $\alpha$ is the frequency skew of oscillator with respect to the nominal frequency, $f_{0}$ of the oscillator.

\subsubsection{Relative Clock Model}

Generally, it is more useful to describe clock characteristics with reference to some other high quality clock source than describing clock as a stand-alone entity. Consequently, the relative clock model, as shown in figure 2.1, is a better choice to model master-slave clock interaction. In this model, $C_{s}$ and $C_{m}$ are periodically spaced vector of time instances and where the period is generally called the clock period which is the inverse of clock frequency. These clocks are related as

$$
C_{s}=\alpha C_{m}+\beta
$$

where $\alpha$ is the relative clock frequency skew which can be expressed as

$$
\alpha=\frac{f_{s}}{f_{m}}
$$

and $\beta$ represents the clock relative time offset or initial difference between master and slave clocks [3].

Time is usually kept using discrete clocks with some finite resolution. This implies that two clocks may show same time but still not synchronized 


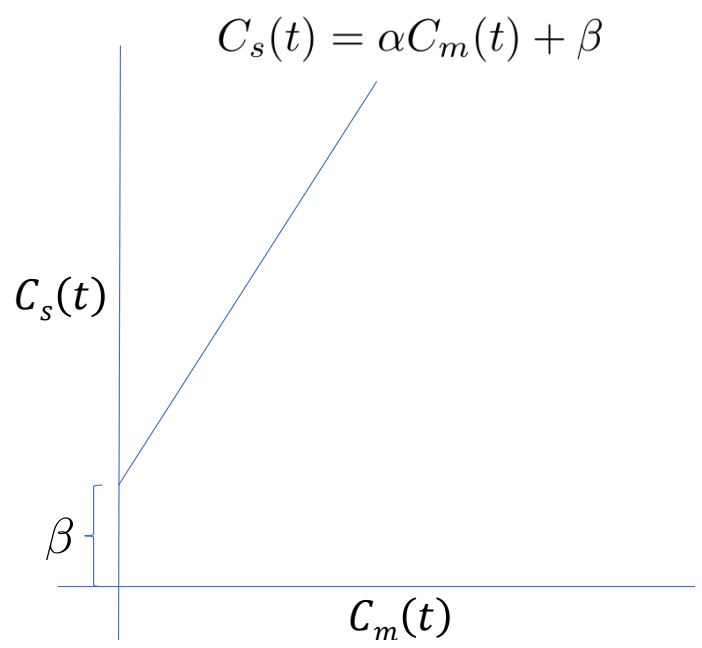

Figure 2.1: Relative Clock Model.

completely due to slight offset between their ticks. This means that, by simply looking at the clock readings, it is not possible to determine $\beta$ to a precision finer than the time resolution of the clocks. At best $\beta$ can represent the coarse time difference between the two clocks. So, an additional clock parameter called clock phase offset, $\phi \in\left(0, T_{s}\right)$ is required to model this slight offset between ticks of the peer clocks. It can be defined as the time duration between the two nearest edges at two clocks, and can be measured in seconds or radians. For example, in case of two wall clocks with resolution of $1 \mathrm{~s}$, the $\phi$ is the fractional time duration between two consecutive clock's second hand tick that occurs at the two clocks. In case of two syntonized clocks i.e. clocks with identical frequencies (say, clock ticks occurring at exactly one second), the clock phase offset, $\phi$ shall remain constant throughout the observation window. On the other hand, the clock phase offset shall vary periodically between two free-running clocks with some constant finite difference in frequencies. So, (2.4) can be re-written to include the effect of clock phase difference, $\phi$ as

$$
C_{s}=\alpha C_{m}+\beta+h\left(t ; f_{d}, \phi\right)
$$

where $h\left(t ; f_{d}, \phi\right)$ is the time varying component, the value of which depends on frequency difference, $f_{d}$ and initial clock phase offset, $\phi$. 


\subsubsection{Synchronization Objective}

In the considered context, the objective is to synchronize slave clock with master's clock. This is done by collecting measurements and using those to estimate clock parameters, $\alpha, \beta$, and $\phi$. We denote the synchronized slave clock as $C_{s, \text { sync }}$. The objective can be formalized as

$$
[\hat{\alpha}, \hat{\beta}, \hat{\phi}]=\min _{\alpha, \beta, \phi} \mathbf{E}\left\{\left|C_{s, \text { sync }}-C_{m}\right|\right\} .
$$

Where $\mathbf{E}$ is the expectation operator. The synchronized clock, $C_{s, s y n c}$ is constructed at slave as

$$
C_{s, s y n c}=\hat{\alpha} C_{m}+\hat{\beta}+h\left(t ; f_{d}, \hat{\phi}\right) .
$$

\subsection{Precision Time Protocol}

This section explains the operation of PTP and some of its terminology and then analyses PTP's estimation capability using the clock model presented in the earlier section.

\subsubsection{Operation}

Figure 2.2 shows the basic message exchange between PTP master and PTP slave nodes. Note that, for simplicity, other initial PTP messages have been omitted.

At the core of the protocol, master at a fixed configured interval sends SYNC message towards the slave node and timestamps the sending time as $t_{1}$ and includes the timestamp value in the SYNC message. However, in general, the precise sending time of the Ethernet frame is not known when the message is created. It is so because Ethernet MAC layer may buffer the message for some unknown duration in its internal queue before writing the frame on the physical medium. Therefore, for better timestamping accuracy, master may optionally send a subsequent FOLLOW_UP message (not shown here) with the exact value of $t_{1}$.

The slave timestamps the receipt of SYNC message as $t_{2}$. The slave, after a known delay at time instance $t_{3}$, sends DELAY_REQ towards master. The master timestamps the receipt of DELAY_REQ as $t_{4}$ and sends the timestamp value back to slave in DELAY_RESP message. At the end of 


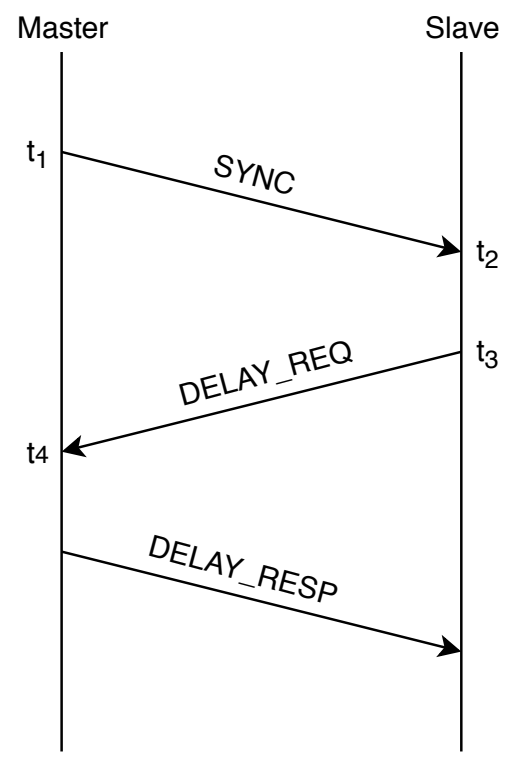

Figure 2.2: A Simplified PTP Timestamp Exchange.

one iteration of message exchange, the slave has the four timestamp values, $\left[t_{1}, t_{2}, t_{3}, t_{4}\right]^{\top}$. The timestamps thus collected are at a known sampling rate which is determined taking into account network conditions and oscillator characteristics.

\subsubsection{Estimation}

The PTP timestamp samples from the sampling window are used by the slave to estimate clock parameters defined in chapter 2 , as follows

1. Relative frequency skew, $\alpha$ is basically the slope or rate of change of slave time with respect to that of master and thus estimated as

$$
\hat{\alpha}_{P T P}=\frac{d C_{m}}{d C_{s}} .
$$

2. The initial clock time difference, $\beta$ is the time difference between master and slave minus the mean path delay, $\Delta$ and thus estimated as

$$
\hat{\beta}_{P T P}=t_{2}-t_{1}-\Delta,
$$

where $\Delta=\frac{\left(t_{2}-t_{1}+t_{4}-t_{3}\right)}{2}$. 
By substituting the above estimated clock parameters in (2.8), slave can generate an estimate of synchronized clock, $C_{s, s y n c}$. Note that the PTP does not observe the phase offset, $\phi$ that may exist between the two clocks, which if observable would have contributed towards the fractional part of $\hat{\beta}_{P T P}$.

\subsubsection{Limitations}

From (2.10), the three error sources that can adversely affect the accuracy of PTP estimates are

1. Network Assymetry: PTP calculates mean path delay by simply averaging the two path delay in each direction. This is based on the underlying assumption that the forward and return path are symmetric (i.e identical path delays). Any asymmetry in the network will introduce a corresponding error in the estimation of mean path delay and thus synchronization precision.

2. Timestamp Inaccuracy: The timestamp inaccuracy arises from the fact that certain PTP packets can be exposed to variable delays due to indeterminate queuing or buffering after they have been timestamped.

3. Limited Timestamp Precision: The driving frequency of the discrete clock limits the highest achievable timestamping resolution and thus introducing a timestamp precision error, $\psi \in\left[0,1 / f_{0}\right)$, where $f_{0}$ is the nominal frequency of the clock. For example, $\psi \in[0,8 \mathrm{~ns})$ timestamp resolution error with a $125 \mathrm{MHz} 1 \mathrm{~Gb}$ Ethernet clock.

Error due to network asymmetry can be overcome through careful network design and provisioning. In other cases, network asymmetry is precisely measured and compensated for in the PTP measurement [15]. To improve timestamp accuracy, PTP unlike its predecessors offers elegant techniques to reduce or eliminate inaccuracies arising out of variable queuing delays through hardware timestamping, FOLLOW_UP message and use of transparent clocks in the networks. Unfortunately PTP as a protocol does not offer any solution to overcome timestamp precision errors. 


\section{Chapter 3}

\section{Proposed Improvement}

The previous chapter (2.2.3) discussed various limitations of PTP that limit the overall achievable time accuracy from the protocol. Among the error sources, timestamp resolution error due to limited timestamp precision is hardest to eliminate as this remains non-observable in most contexts. This problem is relevant for all timestamp exchange based protocols. In case of PTP, It should be noted that only asynchronous timestamps, $t_{2}$ and $t_{4}$ are susceptible to timestamp resolution error. This is so because SYNC and DELAY_REQUEST messages can be synchronized with clock edges through hardware support and consequently eliminating the need of fractional part of $t_{1}$ and $t_{3}$ during measurement. Section 3.1 discusses more about the timestamp resolution error and section 3.2 proposes improvements to both operating modes of PTP viz. two-way and one-way PTP, as means to reduce it and as a result improve PTP's estimations. Each improvement is presented as a protocol with its measurement and estimations models. In the following chapter 4 experiments results are presented demonstrating the effectiveness of the proposed methods.

\subsection{Timestamp Resolution Error or Unknown Clock Phase Offset}

The $t_{2}$ and $t_{4}$ timestamp resolution error improvement can also be seen as relative clock phase offset estimation problem. This is so because if clock phase offset between two node is known along with path delay, the exact time instance when the SYNC or DELAY_REQUEST actually arrives at the 
PTP node can be estimated. In other words, $t_{2}$ and $t_{4}$ can be measured with a resolution higher than permitted by the clock frequency. Mathematically, this can be can be expressed as

$$
\psi=T-\bmod _{T}(\Delta+\phi),
$$

where $\psi$ is the sub-clock timestamp resolution error, $T$ is the clock period, $\bmod _{T}$ is the standard modulo operation over interval $T, \Delta$ is the path delay, and $\phi$ is the relative clock phase offset between the two nodes. Given $\Delta$ and $\phi$, timestamp resolution error $\psi$ can be calculated which if deducted from the individual timestamp values, $t_{2}$ and $t_{4}$, improves their precision.

Unfortunately PTP, in its current form does not provide any estimate of clock phase offset. As a result, timestamp resolution error appears as noise in $t_{2}$ and $t_{4}$ measurement samples and adversely affects time synchronization precision. The worst-case error magnitude is equal to the time duration between two subsequent clock edges, magnitude of which is larger for low frequency clocks. On $125 \mathrm{MHz} 1 \mathrm{~Gb}$ Ethernet clock, this translates to 8 ns worst-case per-hop timestamping error. The error inflates with multiple hops resulting in significant end-to-end time synchronization loss. This is undesirable in telecom use cases such as wireless positioning where every nanosecond error introduces $30 \mathrm{~cm}$ positioning error. Other domains such as power distribution network incur wastage due to loss of synchronization in the network.

\subsubsection{Round Trip Time (RTT) Based Clock Parameter Estimation}

Dwivedi et al (2015) [3] have devised a Round Trip Time (RTT) based clock synchronization method to precisely estimate clock parameters between two wireless nodes without any timestamp exchange. Figure 3.1 shows one such RTT sample. In the paper, RTT measurements have been shown to follow a time varying periodic sawtooth behavior as shown in figure 3.2. This phenomenon results from the relative clock phase offset, $\phi$ and frequency offset, $f_{d}$ between master and slave nodes. The paper also provides several estimators to extract phase and frequency offsets from the RTT sawtooth measurement plot with different degrees of accuracy. 


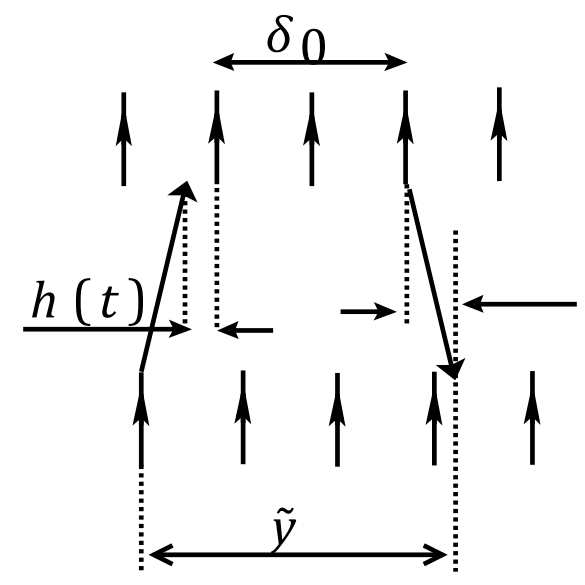

Figure 3.1: Round Trip Time Sample as described in [3]

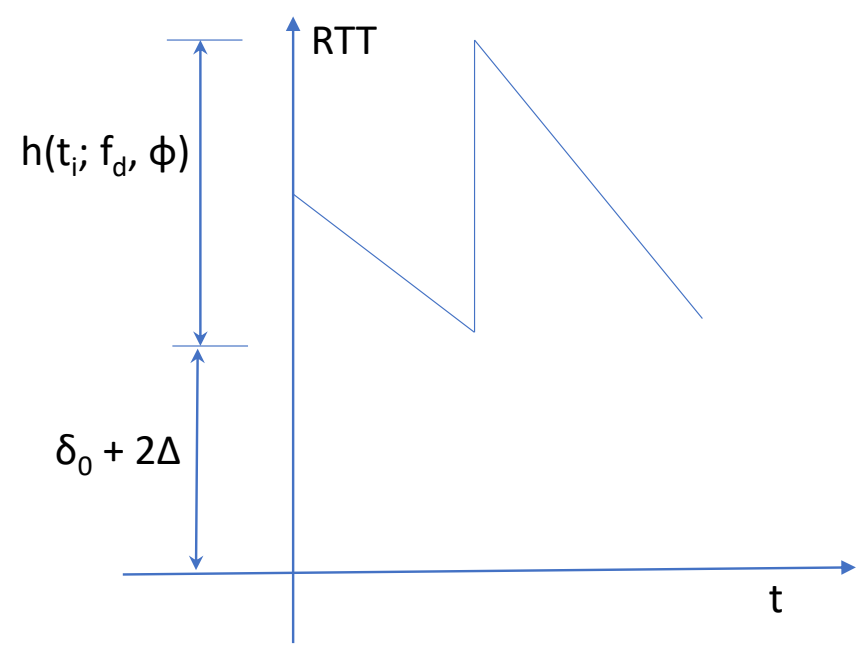

Figure 3.2: Round Trip Time Sawtooth 
Each collected RTT sample at time instant, $t_{i}$ can be mathematically expressed as

$$
y\left(\tilde{t}_{i}\right)=2 \Delta+\delta_{0}+h\left(t_{i} ; f_{d}, \phi\right),
$$

where $\Delta$ is the path delay of PING and RESPONSE signals, and $\delta_{0}$ is the fixed known delay introduced by the receiver of the first PING, and $\phi$ is the sub-clock period phase offset. $h\left(t_{i} ; f_{d}, \phi\right)$ represents the variable time duration between the receipt of PING and next clock edge, the value of which depends upon $\phi$ and $f_{d}$. The paper demonstrates that $h\left(t_{i} ; f_{d}, \phi\right)$ is the time varying component in the equation that imparts the peculiar sawtooth behaviour to RTT samples (Figure 3.2). Modeling this component as a function of $\phi$ and $f_{d}$, allows estimation of both parameters through curve fitting or least squared error techniques. Some of the key features of this method are

1. It does not require syntonized clocks for phase offset measurement.

2. It provides estimates of clock parameters such as phase, frequency offsets, and mean path delay without timestamp exchange.

3. It does not require any specialized hardware except higher resolution time-to-digital converter (TDC) at one of the nodes.

4. It does not require any specialized transport. In the original experiment, ultra wideband (UWB) wireless medium was used to demonstrate its working. 


\subsection{Proposed PTP Improvements}

Although commercial PTP deployments have been successful to achieve synchronization precision of the order of a few hundred nanonseconds but achieving any higher precision is not viable without escalating costs. To enable PTP to estimate sub time period clock phase offset, two methods are proposed that can be seamlessly applied to one-way and two-way PTP. Both these methods employ RTT sawtooth technique for clock phase offset estimation.

\subsubsection{Improved Two-way Precision Time Protocol}

Figure 3.3 illustrates the proposed super-imposed RTT measurement protocol within PTP message exchange. The vertical lines denote PTP master and slave nodes with independent clocks with their clock ticks shown as equidistant short horizontal lines. As nodes track time by counting clock edges, time is most precisely known at these clock edges. In the figure, $t_{s}=t_{0}$ if the set of measurements starts at $t_{s}$.

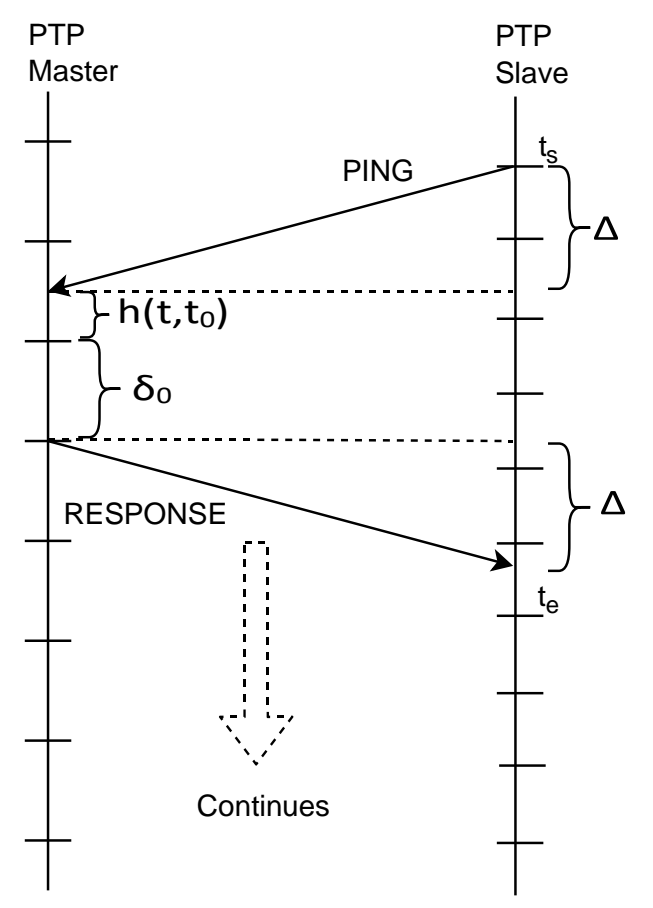

Figure 3.3: RTT Measurement Protocol. 


\section{Protocol}

1. PTP slave sends a PING on positive clock edge and TDC receives the START signal at $t_{s}$

2. On receiving the pulse, PTP master starts the delay generation from subsequent positive edge of its own clock

3. PTP master sends RESPONSE after the delay, $\delta_{0}$ (e.g. one clock cycle in this example).

4. PTP slave receives the RESPONSE, and TDC gets the STOP signal at $t_{e}$ and measures the RTT.

For the above protocol, standard PTP DELAY_REQ and DELAY_RESP messages can be used in place of non-standard PING and RESPONSE messages, respectively.

\section{RTT measurement model}

From Figure 3.3, an RTT sample measured through the high resolution TDC device, in absence of noise, can be mathematically expressed as

$$
y(t)=t_{e}-t_{s}=2 \Delta+\delta_{0}+h\left(t, t_{0}\right),
$$

where $\Delta$ is the path delay of PING and RESPONSE signals, and $\delta_{0}$ is the fixed known delay introduced by the master. $h\left(t, t_{0}\right)$ represents the relative clock phase offset between master and slave clocks at time $t$, which is initialized at time $t=t_{0}$. In other words, the function $h\left(t, t_{0}\right)$ is the variable time duration between the receipt of PING and next clock edge. From $[3], h\left(t, t_{0}\right)$ can be mathematically represented as :

$$
h\left(t, t_{0}\right)=\bmod \left(\frac{1}{2 \pi} \bmod \left(2 \pi f_{d} t+2 \pi f_{s} \phi, 2 \pi\right), \frac{1}{f_{s}}\right),
$$

where mod is the modulo operation, $f_{d}$ and $\phi$ are the frequency and phase offsets between the two clocks respectively, and $f_{s}$ is the frequency of the slave clock. 


\section{RTT Estimation}

The RTT samples, $y(t)$ collected as per the proposed protocol, is expected to exhibit a periodic sawtooth-like waveform as shown in Figure 3.2. From the sawtooth, the following parameters can be estimated

1. The frequency difference, $f_{d}$, which is the frequency of the periodic function $h\left(t, t_{0}\right)$. Using this, the relative frequency skew can be calculated as

$$
\hat{\alpha}_{R T T}=\frac{f_{s}}{f_{m}}=\frac{f_{s}}{f_{s}-f_{d}},
$$

where $f_{s}$ is the PTP slave's clock frequency and is assumed to be known. Note that the sign of $f_{d}$ can be positive or negative in the above equation depending upon whether $f_{s}>f_{m}$ or $f_{s}<f_{m}$ respectively. In case of the former, a rising sawtooth shall be observed and in the latter, a descending edge sawtooth like shown in figure 3.2 is observed.

2. The mean path delay, $\hat{\Delta}$ of equation (3.3).

3. The relative clock phase offset between the two clocks, $\hat{\phi}$ which is the phase of the periodic function $h\left(t, t_{0}\right)$ at time $t=t_{0}$. In other words, $\hat{\phi}$ is the sawtooth's initial phase.

4. Sub-clock period time error, $\hat{\psi} \in\left[0,1 / f_{s}\right)$ is the time difference between slave and master clock edges at the time instance, $t_{0}$. This is calculated as

$$
\hat{\psi}=T_{s}-\bmod _{T_{s}}(\hat{\Delta}+\hat{\phi}),
$$

where $T_{s}$ is the clock period of the PTP slave.

\subsubsection{Improved One-way Precision Time Protocol}

One-way PTP i.e. master clock's periodic one-way signaling in the form of SYNC message towards slave is commonly used to syntonize (or frequency synchronize) the slave clock to that of the master. The $t_{1}$ and $t_{2}$ measurement samples thus collected are then used to estimate the relative frequency skew, $\alpha$ between the two clocks, as seen in equation (2.9). With this method any relative phase offset between the two clocks, if present, remains ignored and contributes towards an error in relative frequency skew estimation. Alternatively, the section below presents a method which provides additional 
estimates of relative clock phase offset between the two clocks enabling finer frequency synchronization of the two clocks.

\section{Protocol}

1. Master, at most precisely measured time instance $t_{1}$ sends the SYNC signal.

2. On receiving the SYNC signal, slave starts the delay generation from subsequent positive edge of its own clock

3. Slave after the delay, $\delta_{0}$ (e.g. two clock cycles in this example) timestamps the instance as $t_{2}$ with highest precision.

4. Slave computes the time difference, $t_{2}-t_{1}$.

5. The process repeats at a certain rate which depends on the frequency difference between the two clocks.

\section{Measurement Model}

The time difference samples at slave, in absence of noise, can be expressed through the following general equation. Figure 3.4 shows one such measurement sample.

$$
y(t)=t_{2}-t_{1}=(\alpha-1) t_{1}+\beta+\Delta+\delta_{0}+g\left(t, t_{0}\right),
$$

where $\alpha$ is the relative frequency skew i.e $f_{s} / f_{m}, \beta$ is the time offset between the two clocks, $\Delta$ is the propagation delay of the signal, $\delta_{0}$ is the fixed delay introduced at slave, and $g\left(t, t_{0}\right)$ is the relative clock phase offset at time $t$ (similar to the time varying component that imparts sawtooth behaviour to RTT samples, refer 3.2), which is initialized at $t=t_{0}$. Taking a cue from [3], $g\left(t, t_{0}\right)$ can also be mathematically represented as:

$$
g\left(t, t_{0}\right)=\bmod \left(\frac{1}{2 \pi} \bmod \left(2 * 2 \pi f_{d} t+2 \pi f_{s} \phi, 2 \pi\right), \frac{1}{f_{s}}\right),
$$

where $f_{d}$ and $\phi$ are the frequency and phase offsets between the two clocks respectively and $f_{s}$ is the frequency of the slave clock. 


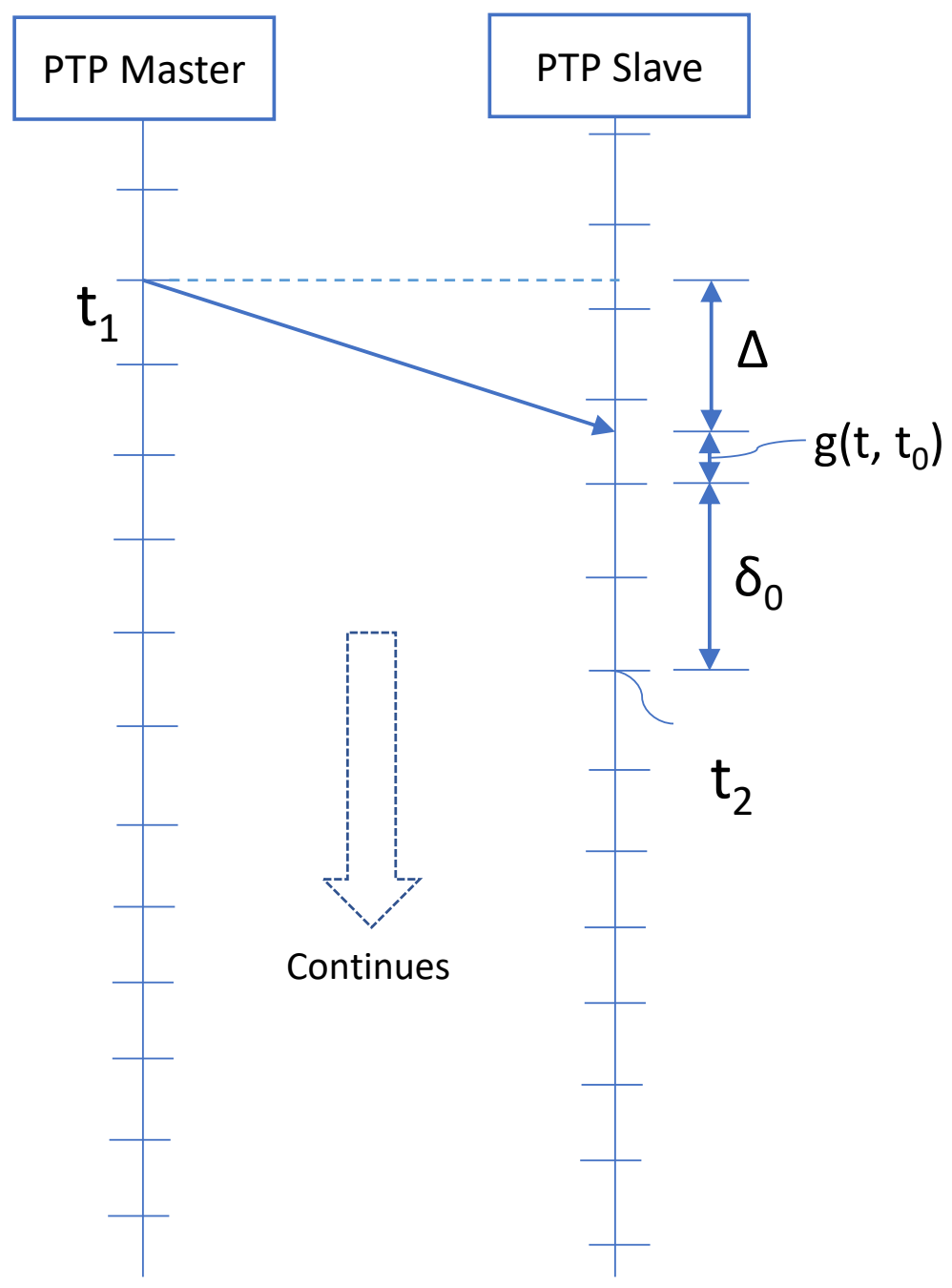

Figure 3.4: Frequency and Phase Estimation through One-Way PTP. 


\section{Clock Parameter Estimation}

The time samples, $y(t)$ collected as per the above protocol shall exhibit a rising $(\alpha>1)$ or tapering $(\alpha<1)$ sawtooth waveform. Using equation (3.7) as the model function and applying curve fitting technique such as least square, the following clock parameters can be estimated

1. The relative clock phase offset between the two clocks, $\hat{\phi}$ is the phase of the periodic function $g\left(t, t_{0}\right)$ at time $t=t_{0}$.

2. The relative frequency skew, $\hat{\alpha}$

3. The absolute frequency offset, $f_{d}$

The last two parameters allow estimation of absolute frequencies of master and slave nodes as follows.

$$
\begin{gathered}
f_{d}=f_{m}-f_{s} \\
\hat{\alpha}=\frac{f_{s}}{f_{m}}
\end{gathered}
$$

Substituting (3.9) into (3.10),

$$
f_{m}=\frac{f_{d}}{1-\hat{\alpha}}
$$

and

$$
f_{s}=\hat{\alpha} f_{m}
$$

This is a noteworthy capability of the proposed method over PTP. This is so because the normal PTP or for that matter any timestamp based sync protocol, only provides means to estimate the ratio of frequencies. 


\section{Chapter 4}

\section{Simulations and Results}

Matlab based simulations were developed to validate and to evaluate performance of the proposed protocol improvements from chapter 3. The simulations implement measurement and estimation models for both one-way and two-way PTP improvement protocols (sections 3.2.1 and 3.2.2).

\subsection{Simulation Methodology}

In the simulations, two free running independent clocks with nominal frequency, $f_{0}=10 \mathrm{MHz}$ are modeled representing PTP master and slave nodes, respectively. Periodic RTT, $\left(t_{4}-t_{1}\right)$ and one-way, $\left(t_{2}-t_{1}\right)$ measurement samples were collected for a configured sampling window duration for different values of $f_{d}$ and transmission noise. On the collected samples, the measurement model as defined in equations (3.3) and (3.7) were used as hypothesis function to estimate clock parameters using least squares regression method. For 'Improved Two-way PTP' scenario (section 3.2.1), the following parameters were estimated:

1. Relative frequency skew values from normal PTP measurement and RTT sawtooth $\left(\hat{\alpha}_{P T P}\right.$ and $\hat{\alpha}_{R T T}$ respectively) which were further combined together to produce an improved estimate of frequency skew, $\hat{\alpha}_{i m p}$.

2. Mean path delay $(\hat{\Delta})$.

3. Relative phase offset, $\hat{\phi}$ which is further used to determine sub-clock period time error $(\hat{\psi})$ using equation $(3.6)$. 
4. Time offset $\hat{\beta}_{i m p}$ by subtracting $\hat{\psi}$ from $\hat{\beta}_{P T P}$, time offset estimate from normal PTP. $\hat{\beta}_{i m p}$ is the improved estimate of time offset with sub-clock period precision.

For 'Improved One-way PTP' scenario (section 3.2.2), only parameters 1) and 3 ) from the above list were estimated (i.e. $\hat{\alpha}_{i m p}$ and $\hat{\phi}$ ). In both simulated scenarios, the respective clock parameter estimates were used by the slave node to generate the time-synchronized or phase-synchronized clock (defined in section 1.3) replica, $C_{s, \text { sync }}$ aligned to that of the master clock, $C_{m}$ ) using two approaches. First, by using normal PTP approach utilizing just $\hat{\alpha}_{P T P}$ and $\hat{\beta}_{P T P}$ estimates (See 2.2.2). And second, by using normal PTP estimates combined with estimates from the sawtooth i.e. based on $\hat{\alpha}_{i m p}$ and $\hat{\beta}_{i m p}$. To measure the accuracy of the generated slave clock, the sync error was calculated as the absolute difference between the master and generated synchronized slave clock i.e (sync_err $\left.=\left|C_{m}-C_{s, \text { sync }}\right|\right)$. The sync error values over 100 iterations were collected for three different values of relative frequency offset, $f_{d}$ and two different values of Gaussian transmission noise standard deviation (simulating path delay variation).

\subsection{Simulation Configuration Parameters}

To simulate real world conditions, following parameters are configurable in the simulations:

1. Initial time offset, $\beta$ in seconds to account for different start times of the respective clocks

2. Initial relative phase offset, $\phi$ in seconds to account for sub-clock period time difference between the adjacent edge of the two clocks.

3. Relative frequency offset, $f_{d}$ in $\mathrm{Hz}$ to account for the effect of inherent frequency deviation in oscillator from its nominal frequency.

4. Sampling rate or number of measurement samples generated per second, $f_{\text {sampling }}$. For the experiments, this was fixed to $100 \mathrm{~K}$ samples/sec.

5. Sampling window duration in seconds. This was fixed to 0.01 seconds. 
6. Zero mean random transmission noise added to one-way message path delay, $\Delta$ to simulate path delay variation. For the experiments, this was randomized with different standard deviation values.

7. Zero mean random jitter noise to simulate clock edge jitter which appears as noise in timestamps. For the experiments this was randomized with a fixed standard deviation of 2 ns.

\subsection{Results and Analysis - Improved Two- way PTP}

Figures $4.1,4.2,4.3,4.4,4.5$, and 4.6 show the sync-error observed for different values of initial frequency difference between clocks and packet delay variations during PTP timestamp exchange. In all cases, the peculiar sawtooth pattern in RTT measurements was observed. The following is inferred from the results:

1. As expected, RTT measurements were observed to follow sawtooth waveform with a frequency approximately equal to that of the frequency difference between the two clocks, conforming to our hypothesis function for RTT.

2. After combining PTP clock parameter estimates (section 2.2.2) with clock parameter estimates from sawtooth (section 3.2.1), the synchronization error was observed to be consistently lower than when pure PTP estimates were used to generate synchronized slave clock.

3. As $f_{d}$ was increased from $100 \mathrm{~Hz}$ to $200 \mathrm{~Hz}$, the variance in sync errors for both approaches were observed to increase. This is expected due to limited precision in accurately estimating relative frequency skew $\alpha$ with large frequency deviation. In a typical time sync set-up, several rounds of periodic clock corrections are employed to reduce the effect of limited precision of estimates.

4. As path delay standard deviation increased from $5 \mathrm{~ns}$ to $10 \mathrm{~ns}$, the sawtooth transition expectantly got less sharper due to uncertainty in packet timestamping around clock edges. This introduces loss of accuracy in phase estimation and consequently the sync error was observed 
to spike for certain samples. But in all cases, sync-error seen with the improved protocol was significantly lower than pure PTP.

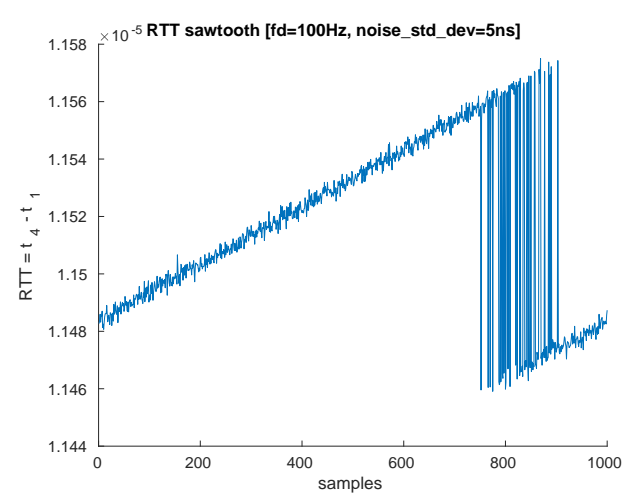

(a) $y=t_{4}-t_{1}$

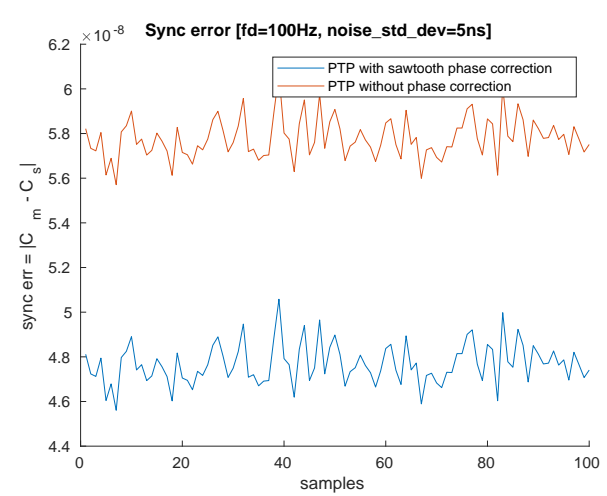

(b) $y=\left|C_{m}-C_{s}\right|$

Figure 4.1: Two-way sync error $\left[f_{d}=100 H z\right.$, path delay std_dev $\left.=5 n s\right]$

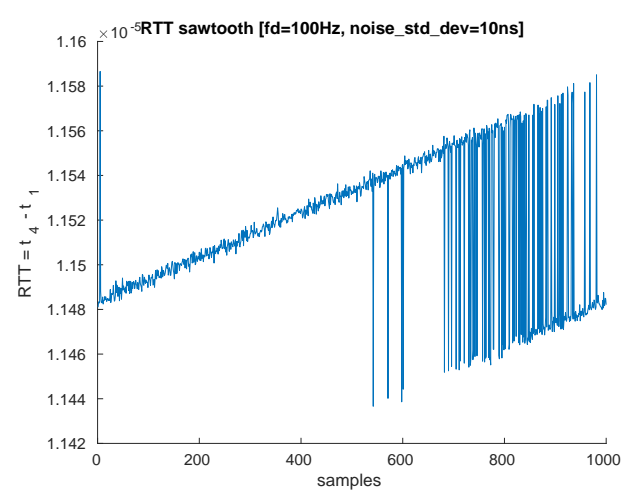

(a) $y=t_{4}-t_{1}$

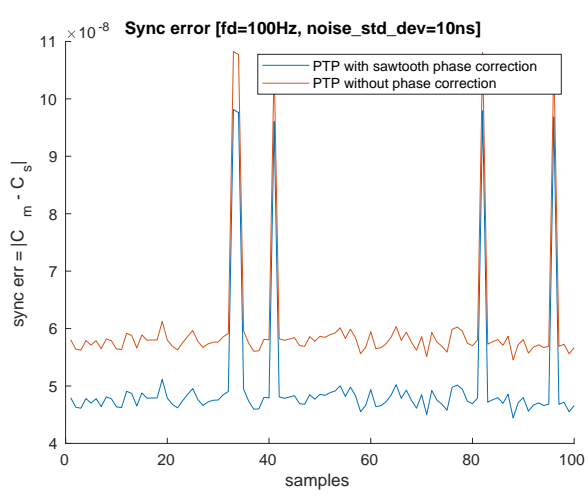

(b) $y=\left|C_{m}-C_{s}\right|$

Figure 4.2: Two-way sync error $\left[f_{d}=100 H z\right.$, path delay std_dev $\left.=10 \mathrm{~ns}\right]$ 


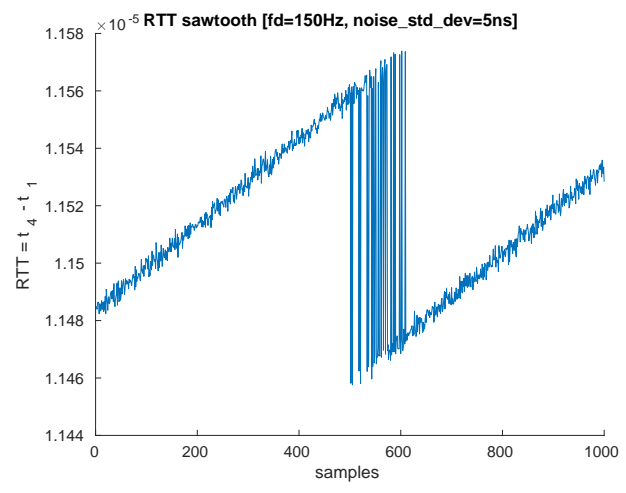

(a) $y=t_{4}-t_{1}$

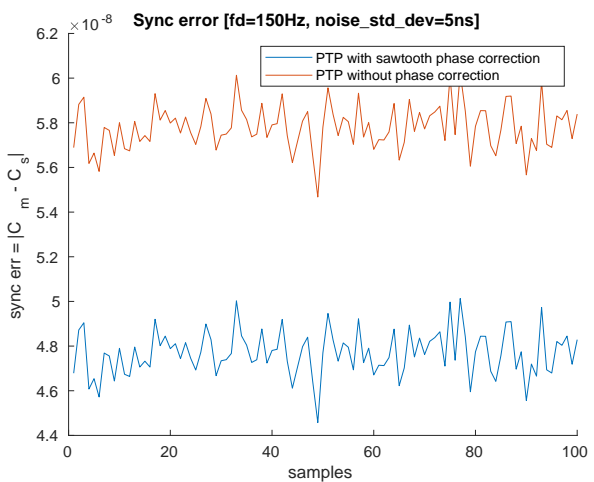

(b) $y=\left|C_{m}-C_{s}\right|$

Figure 4.3: Two-way sync error $\left[f_{d}=150 \mathrm{~Hz}\right.$, path delay std_dev $\left.=5 \mathrm{~ns}\right]$

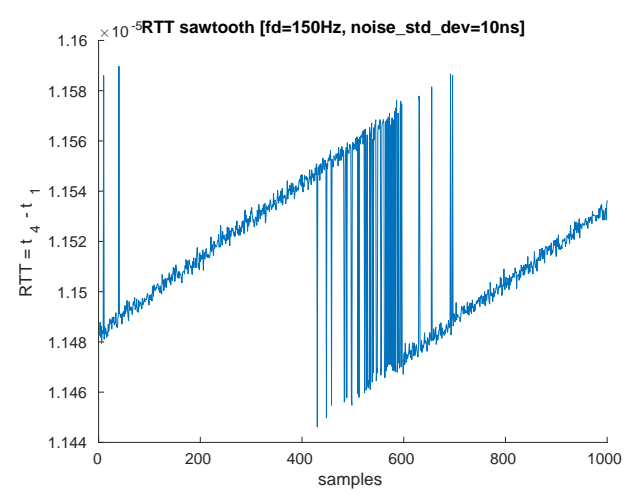

(a) $y=t_{4}-t_{1}$

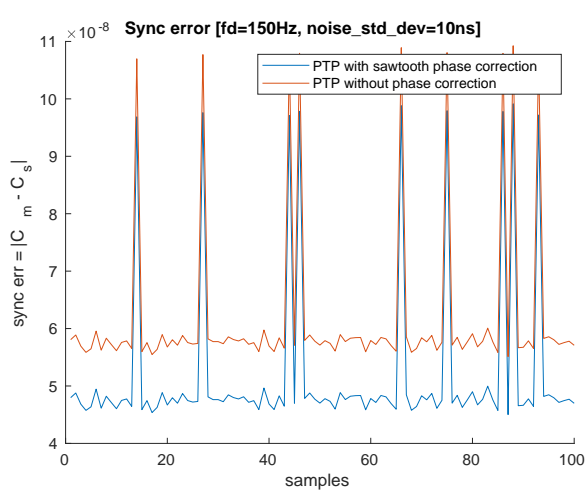

(b) $y=\left|C_{m}-C_{s}\right|$

Figure 4.4: Two-way sync error $\left[f_{d}=150 \mathrm{~Hz}\right.$, path delay std_dev $\left.=10 \mathrm{~ns}\right]$ 


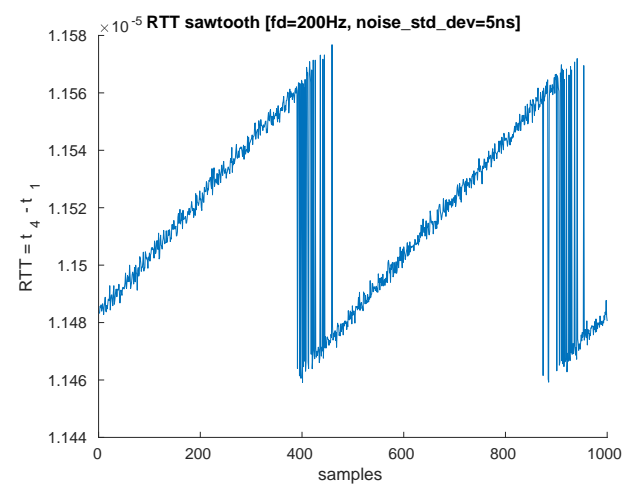

(a) $y=t_{4}-t_{1}$

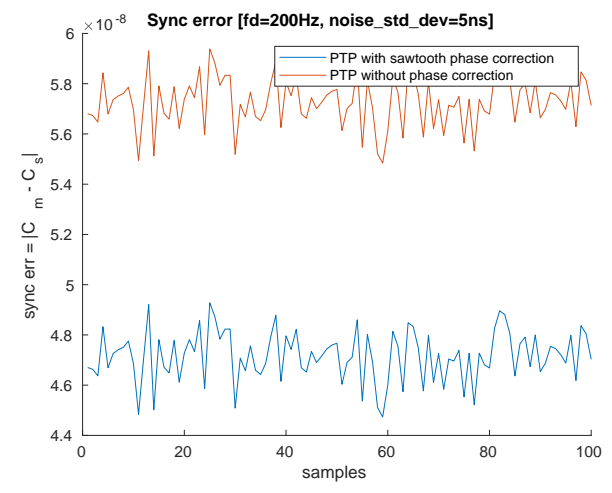

(b) $y=\left|C_{m}-C_{s}\right|$

Figure 4.5: Two-way sync error $\left[f_{d}=200 \mathrm{~Hz}\right.$, path delay std_dev $\left.=5 n s\right]$

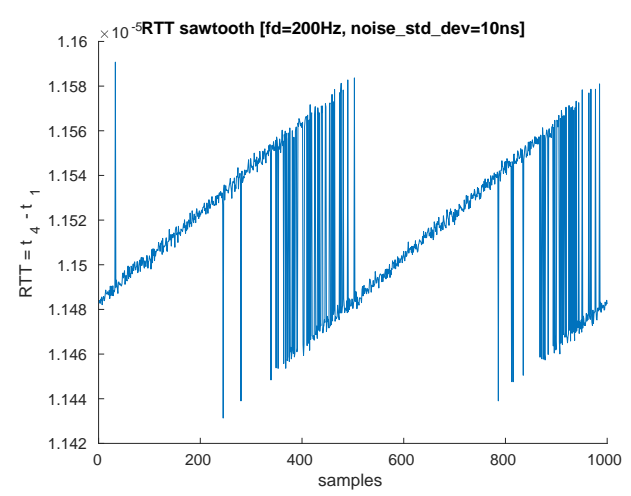

(a) $y=t_{4}-t_{1}$

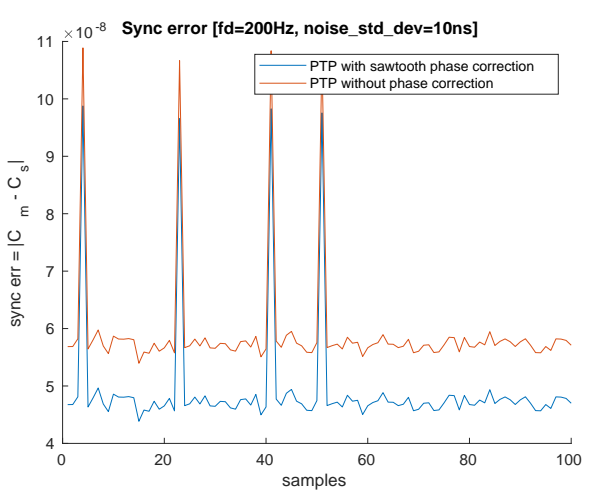

(b) $y=\left|C_{m}-C_{s}\right|$

Figure 4.6: Two-way sync error $\left[f_{d}=200 H z\right.$, path delay std_dev $\left.=10 n s\right]$ 


\subsection{Results and Analysis - Improved One-way PTP}

Figures 4.7, 4.8, 4.9, 4.10, 4.11, and 4.12 show the sync-error observed for different values of initial frequency difference between clocks and packet delay variations during one-way PTP timestamp transfer. Some key observations are as follows:

1. The peculiar ramped sawtooth was observed in all cases with the frequency approximately twice that of the frequency difference between the two clocks. This is unlike the sawtooth observed in case of twoway PTP where the sawtooth frequency was approximately same as the frequency difference between the two clocks.

2. The sawtooth was seen to have a downward ramp due to a positive value of $f_{d}$ (i.e. $f_{m}>f_{s}$ ).

3. PTP estimation combined with sawtooth phase correction generally showed significantly lower synchronization error compared to normal one-way PTP.

4. As $f_{d}$ was increased from $100 \mathrm{~Hz}$ to $200 \mathrm{~Hz}$, the sync error variance, expectantly, was observed to increase for both approaches.

5. As path delay standard deviation increased from $5 \mathrm{~ns}$ to $10 \mathrm{~ns}$, the sawtooth transition expectantly got less sharper introducing some slight loss of accuracy in phase estimation and thereby slightly increasing the sync error. 


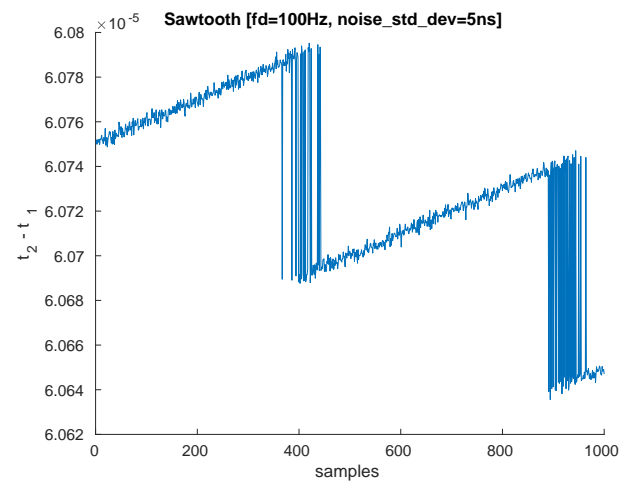

(a) $y=t_{2}-t_{1}$

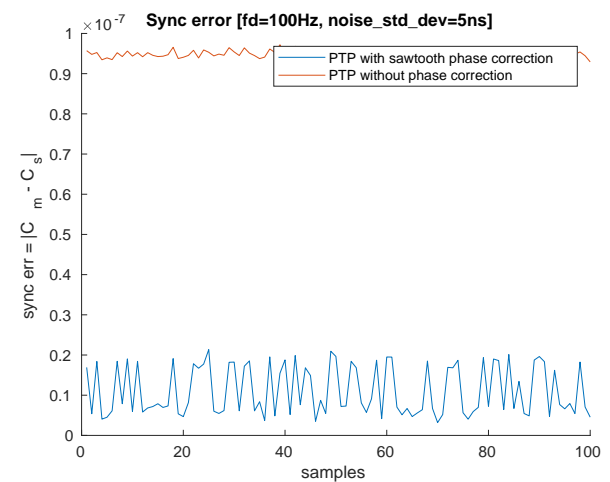

(b) $y=\left|C_{m}-C s\right|$

Figure 4.7: One-way sync error $\left[f_{d}=100 \mathrm{~Hz}\right.$, path delay std_dev $\left.=5 \mathrm{~ns}\right]$

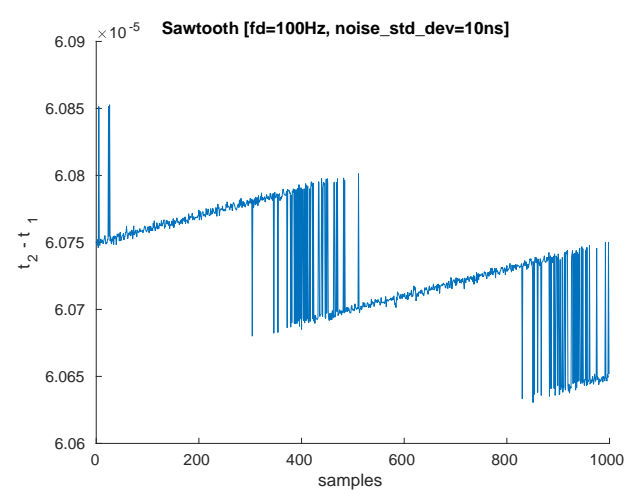

(a) $y=t_{2}-t_{1}$

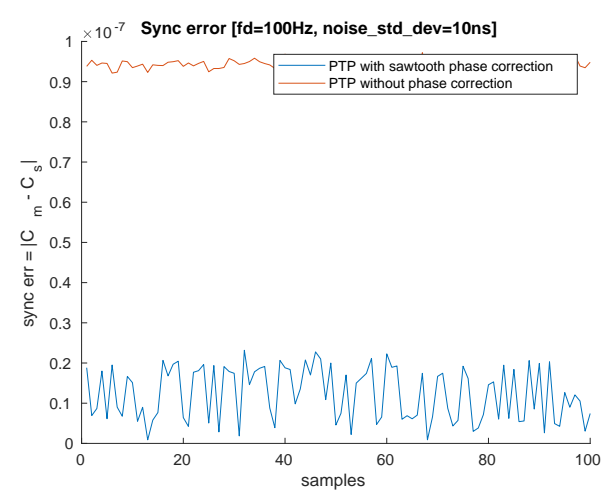

(b) $y=\left|C_{m}-C s\right|$

Figure 4.8: One-way sync error $\left[f_{d}=100 \mathrm{~Hz}\right.$, path delay std_dev $\left.=10 \mathrm{~ns}\right]$ 


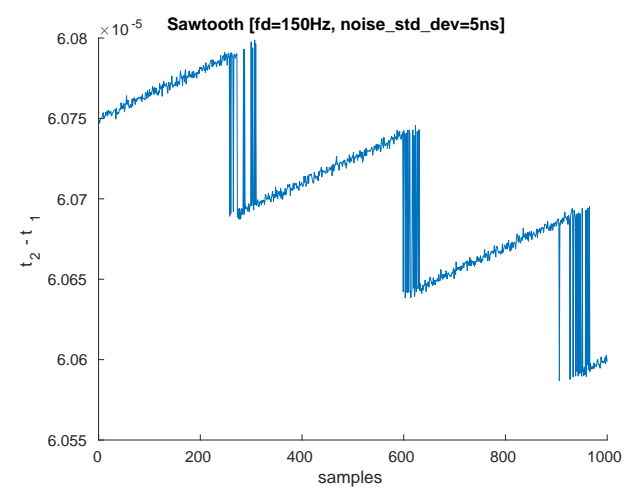

(a) $y=t_{2}-t_{1}$

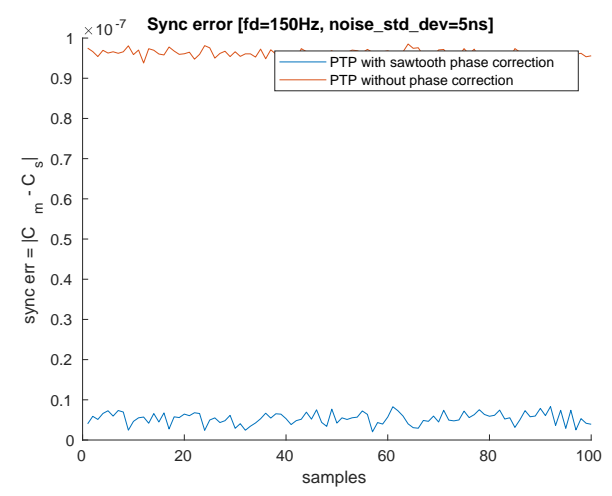

(b) $y=\left|C_{m}-C s\right|$

Figure 4.9: One-way sync error $\left[f_{d}=150 \mathrm{~Hz}\right.$, path delay std_dev $\left.=5 n s\right]$

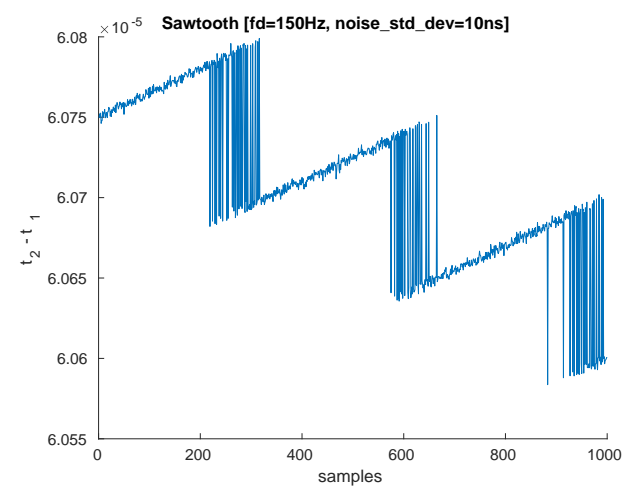

(a) $y=t_{2}-t_{1}$

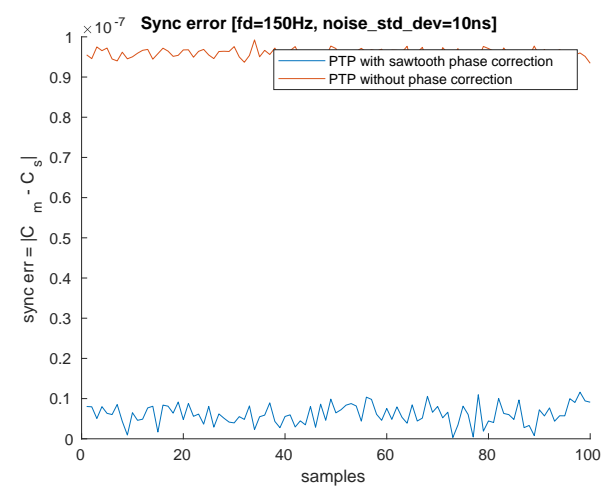

(b) $y=\left|C_{m}-C s\right|$

Figure 4.10: One-way sync error $\left[f_{d}=150 \mathrm{~Hz}\right.$, path delay std_dev $\left.=10 \mathrm{~ns}\right]$ 


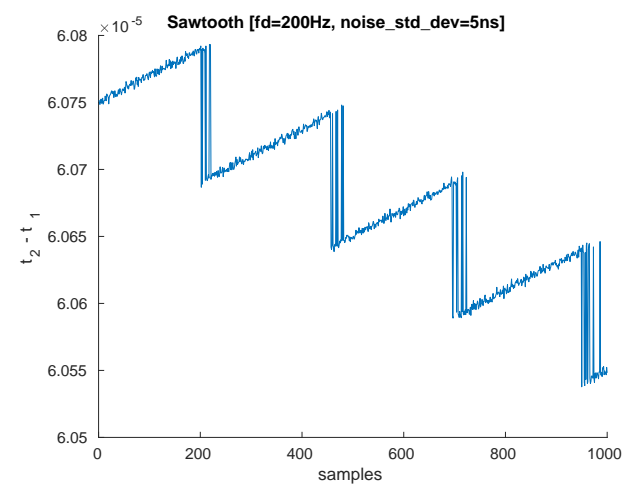

(a) $y=t_{2}-t_{1}$

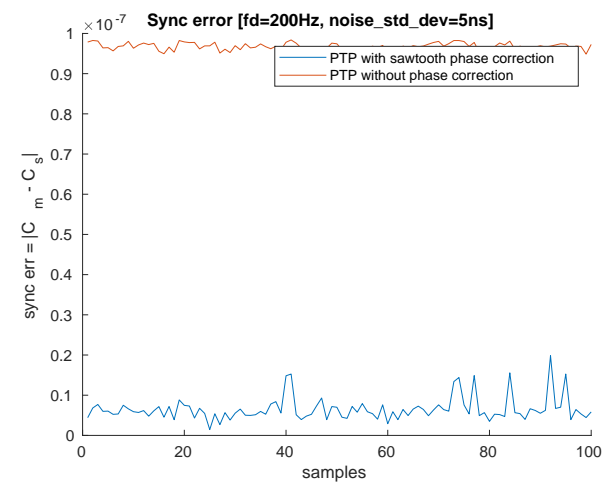

(b) $y=\left|C_{m}-C s\right|$

Figure 4.11: One-way sync error $\left[f_{d}=200 H z\right.$, path delay std_dev $\left.=5 n s\right]$

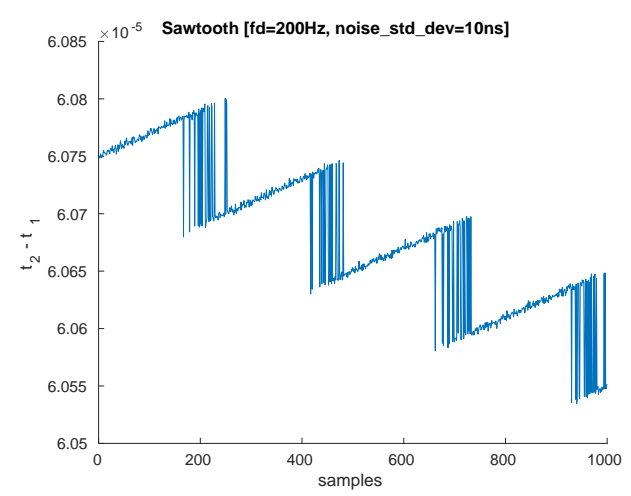

(a) $y=t_{2}-t_{1}$

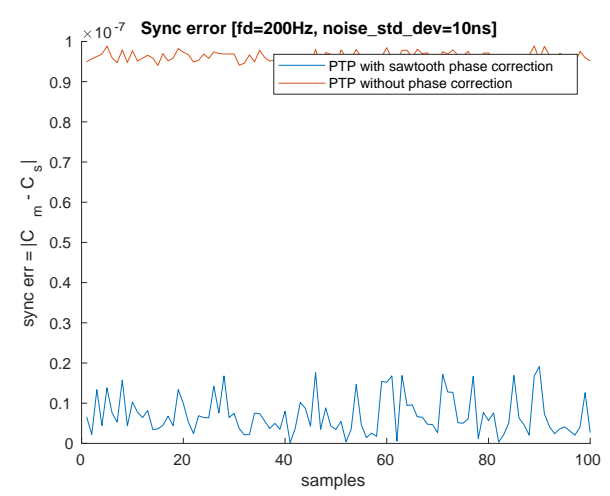

(b) $y=\left|C_{m}-C s\right|$

Figure 4.12: One-way sync error $\left[f_{d}=200 H z\right.$, path delay std_dev $\left.=10 \mathrm{~ns}\right]$ 


\section{Chapter 5}

\section{Comparison with White Rabbit}

\subsection{White Rabbit}

CERN's Large Hadron Collider (LHC) [10] requires extremely high (subnanosecond) precision time distribution among its accelerator sites to synchronize accelerator operation and control, and to chronologically order the events occurring inside the accelerator. Recognizing PTP's inablity to deliver such high precision in a wide geographical area, CERN's White Rabbit [4] project has introduced additional capability into PTP, in the form optional PTP profile, to precisely measure link assymetry and sub clock period phase offset, and use them to improve PTP's timing accuracy (see figure 5.1). In other words, the project attempts to plug the most dominant PTP error sources. This new protocol enhancements is targeted exclusively for Synchronous Ethernert (SyncE) based networks. While the SyncE at physical layer provides frequency synchronization between two nodes, WR uses hardware phase detectors in the form of phase linked loops (PLL) to measure relative phase difference between the two clocks. The measured phase difference is then used by respective nodes to improve the precision of asynchronous PTP timestamps $\left(t_{2}\right.$ and $\left.t_{4}\right)$. Although WR has managed to achieve incredible results but its widespread adoption is expected to have some hurdles due to its dependency on specific hardware and transport technology. For instance, WR measures relative phase offset through dedicated round trip signalling and PLL implemented in hardware. This implies that all switches including intermediate switches within a network should be WR compliant to achieve the promised precision. Furthermore, assuming master 
and slave clocks to be syntonized is a strong assumption for several networks. In such networks, frequency and time synchronization are performed through the same method, such as PTP. And therefore, WR's phase detector method may not be feasible in such scenarios. Finally, WR's enhancement is available only with SyncE as the underlying transport protocol. This means legacy networks will require major overhauls to benefit from WR's methods.

\subsection{RTT based PTP improvement}

On the other hand, the proposed RTT based PTP improvements in this report utilizes a natural phenomenon between two free running clocks to provide estimates of clock parameters including relative phase offset and requires only limited extra support from hardware. For instance, Improved Two-way PTP (3.2.1) may require support for high resolution Time-to-Digital converter (TDC) device on the master side to count time interval with high precision if not already available. Improved One-way (see 3.2.2) does not require any extra hardware support. Both improvements do not have any dependency on any specific transport technology. This implies that benefits of the improved estimates can be availed in existing PTP deployments with a simple software upgrade on PTP compliant switches. As the proposed improvements only provide 'estimates' of clock parameters, the accuracy of these is expected to be lower than 'measurements' resulting out of WR's method. 
a) White Rabbit Link Model

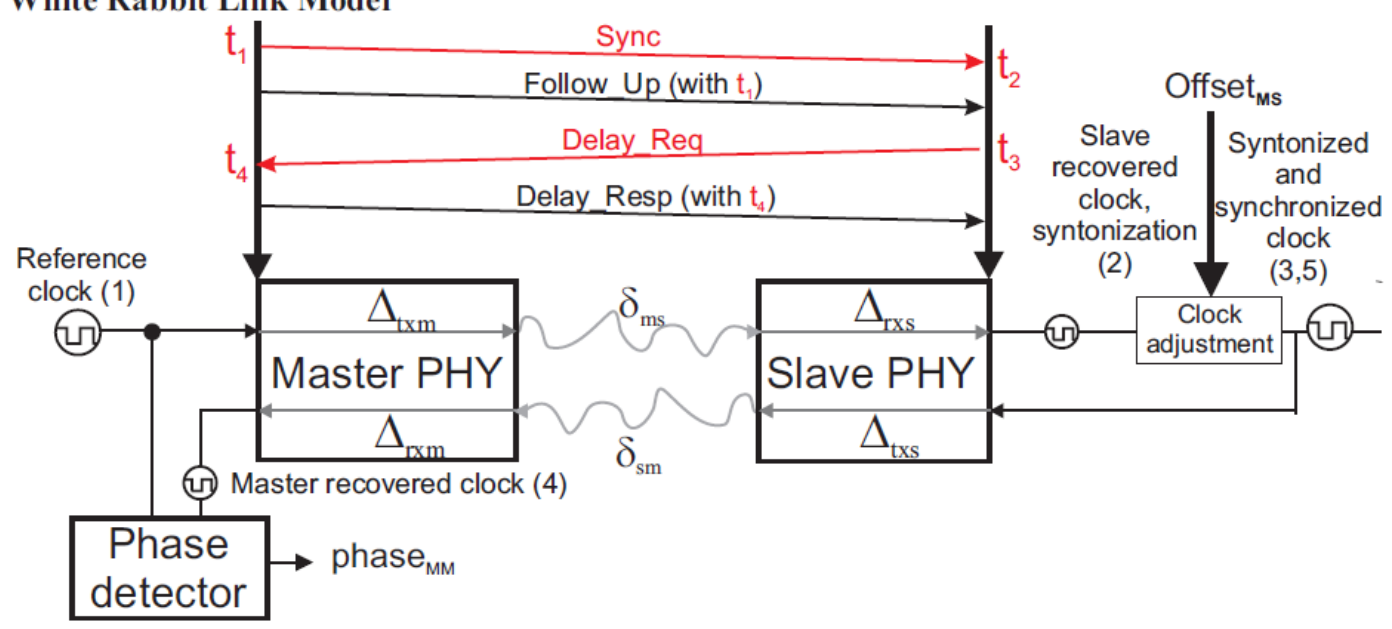

b) White Rabbit synchronization and syntonization scheme

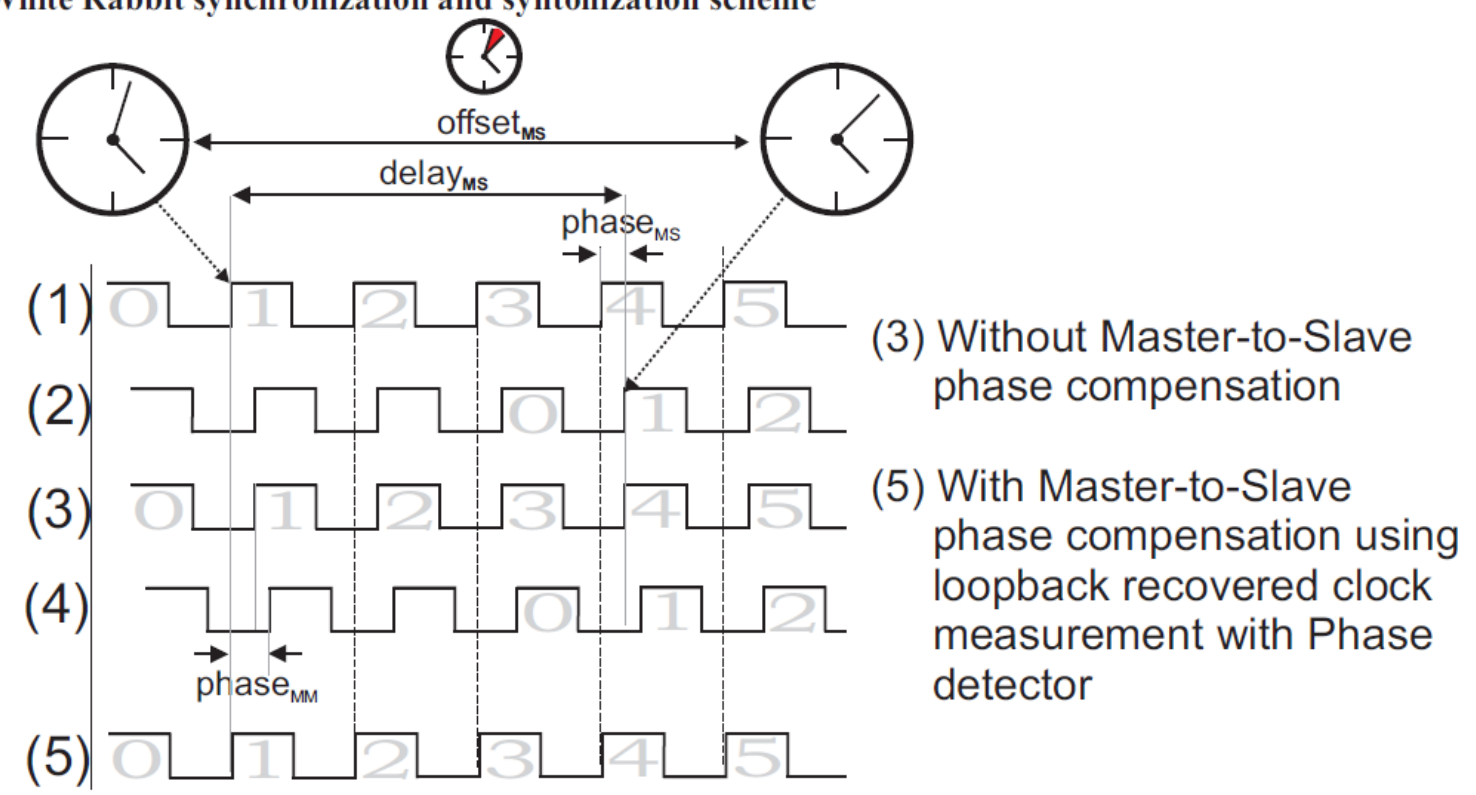

Figure 5.1: White Rabbit Synchronization Scheme [4] 


\section{Chapter 6}

\section{Discussion and Conclusion}

As discussed in section 2.2.3, PTP's clock synchronization accuracy depends majorly on the accuracy and precision of timestamp samples. In real world networks, timestamping devices use discrete clocks to timestamp events. This implies a finite uncertainty in event timestamping as actual events may occur between two clock edges of the discrete clock. The magnitude of this uncertainty depends on the clocks' frequency and phase differences.

The formal analysis of PTP (2.2) shows that it does not detect clock phase difference and therefore timestamp resolution error arising due to clocks' relative phase offset ends up in PTP's $t_{2}$ and $t_{4}$ samples as measurement noise. To overcome this inherent PTP shortcoming, the two-way (3.2.1) and one-way (3.2.2) PTP improvements that enable estimation of clocks' phase difference estimation along side normal PTP estimates, were proposed, analysed, and simulated to demonstrate their effectiveness.

It is noteworthy that the proposed methods use a well-defined signal model, in the form of the sawtooth-like waveform, arising out of a natural phenomenon between two free-running clocks. This can have additional usage beyond those discussed in this report. For instance, the unique sawtooth pattern offers efficient filtering means to select best PTP packets for clock synchronization, thereby reducing noisy PTP measurement samples. Furthermore, as the sawtooth is essentially the result of frequency and phase difference between the clocks, an absence of it could be interpreted as an evidence of perfect or near perfect synchrony. As mentioned in section 3.2.2, the proposed methods also provide means to estimate absolute values of the master and slave clocks' frequencies unlike PTP which only provides frequency ratios. In other words, a node by simply listening to incoming timestamped 
signals, can precisely estimate the absolute clock frequency of the remote sender. This may have wide ranging use cases, say in lab instruments used for measuring frequencies of devices.

PTP improvements using phase correction is not a new concept. It is one of the core ideas that CERN's White Rabbit project [4] has utilized to achieve sub-nanosecond precision. What distinguishes the proposed RTT based PTP enhancement is the method of phase estimation which does not require any necessary hardware or specific transport technology. This allows existing PTP deployments to benefit from the improved accuracy without any major infrastructure impact. Due to the simplicity of the RTT protocol, its integration with PTP is straightforward. To maintain backward compatibility with PTP, the RTT measurements can also be introduced as an optional PTP profile that slave can negotiate with master during initial PTP handshake.

One potential concern around RTT based estimation could be that high sampling rate $\left(>2 f_{d}\right)$ is needed to observe the sawtooth, if the frequency difference between slave and master is high. But this may not be a major problem. To overcome the problem, synchronization procedure between two clocks could be performed in two phases - coarse sync with standard PTP which can bring down the frequency error to manageable level, and finer sync where RTT sawtooth can be formed at relatively low sampling rate.

The results [4] from the simulations demonstrate that the proposed supplemental protocols provides valuable clock parameter estimates related to frequency and phase information which when combined with standard PTP, significantly improves clock synchronization accuracy. Further, the proposed methods can be seamlessly applied to PTP without requiring any major changes in the existing protocol exchange and hardware infrastructure and therefore can be seen as an inexpensive but effective improvement. The improved clock synchronization improves coordinated operations of network end-points resulting in efficient usage of resources and energy savings in several domains such as telecommunications, financial networks, scientific networks, and power distribution grids. 


\section{Bibliography}

[1] M. R. Gholami, S. Gezici, and E. G. Strom, "Tdoa based positioning in the presence of unknown clock skew," IEEE Transactions on Communications, vol. 61, no. 6, pp. 2522-2534, 2013.

[2] Ericsson, "Network synchronization : A key component of ericsson's evolved ip network solution," no. 5/287 01-FGB 101686 Rev B.

[3] S. Dwivedi, A. De Angelis, D. Zachariah, and P. Händel, "Joint ranging and clock parameter estimation by wireless round trip time measurements," IEEE Journal on Selected Areas in Communications, vol. 33, no. 11, pp. 2379-2390, 2015.

[4] M. Lipiński, T. Włostowski, J. Serrano, and P. Alvarez, "White rabbit: A PTP application for robust sub-nanosecond synchronization," in Precision Clock Synchronization for Measurement Control and Communication (ISPCS), 2011 International IEEE Symposium on. IEEE, 2011, pp. 25-30.

[5] R. Bajaj, S. L. Ranaweera, and D. P. Agrawal, "Gps: location-tracking technology," Computer, vol. 35, no. 4, pp. 92-94, 2002.

[6] D. Mills, J. Martin, J. Burbank, and W. Kasch, "Rfc 5905: Network time protocol version 4: Protocol and algorithms specification," Internet Engineering Task Force, 2010.

[7] "Ieee standard for a precision clock synchronization protocol for networked measurement and control systems - redline," IEEE Std 15882008 (Revision of IEEE Std 1588-2002) - Redline, pp. 1-300, July 2008. 
[8] D. Bladsjö, M. Hogan, and S. Ruffini, "Synchronization aspects in lte small cells," IEEE Communications Magazine, vol. 51, no. 9, pp. 70-77, 2013.

[9] H. Li, L. Han, R. Duan, and G. M. Garner, "Analysis of the synchronization requirements of $5 \mathrm{~g}$ and corresponding solutions," IEEE Communications Standards Magazine, vol. 1, no. 1, pp. 52-58, 2017.

[10] CERN. (1999) Cern homepage. [Online]. Available: https://home.cern/science/accelerators/large-hadron-collider

[11] G. DeCandia, D. Hastorun, M. Jampani, G. Kakulapati, A. Lakshman, A. Pilchin, S. Sivasubramanian, P. Vosshall, and W. Vogels, "Dynamo: amazon's highly available key-value store," ACM SIGOPS operating systems review, vol. 41, no. 6, pp. 205-220, 2007.

[12] D. L. Mills, Computer network time synchronization: the network time protocol on earth and in space. CRC press, 2016.

[13] ITU-T, "G.8275.1 : Precision time protocol telecom profile for phase/time synchronization with full timing support from the network," 2020. [Online]. Available: https://www.itu.int/rec/T-RECG.8275.1-202003-I/en

[14] I. Skog and P. Handel, "Time synchronization errors in loosely coupled gps-aided inertial navigation systems," IEEE Transactions on Intelligent Transportation Systems, vol. 12, no. 4, pp. 1014-1023, 2011.

[15] R. Exel, "Mitigation of asymmetric link delays in ieee 1588 clock synchronization systems," IEEE Communications Letters, vol. 18, no. 3, pp. 507-510, 2014. 
TRITA-EECS-EX-2020:865

www.kth.se 